\title{
Radiative properties of a plasma moving across a magnetic field. I: Theoretical analysis
}

\author{
Robert Roussel-Dupré \\ Space Science and Technology Division, Los Alamos National Laboratory, Los Alamos, New Mexico \\ 87545 \\ Ronald H. Miller \\ Space Physics Research Laboratory, Department of Atmospheric, Oceanic and Space Sciences, \\ The University of Michigan, Ann Arbor, Michigan 48109-2143
}

(Received 25 July 1991; accepted 8 January 1993)

\begin{abstract}
The early-time evolution of plasmas moving across a background magnetic field is addressed with a two-dimensional model in which a plasma cloud is assumed to have formed instantaneously with a velocity across a uniform background magnetic field and with a Gaussian density profile in the two dimensions perpendicular to the direction of motion. This model treats both the dynamics associated with the formation of a polarization field and the generation and propagation of electromagnetic waves. In general, the results indicate that, to zeroth order, the plasma cloud behaves like a large dipole antenna oriented in the direction of the polarization field which oscillates at frequencies defined by the normal mode of the system. The magnitude of the radiation field and the amount of plasma momentum and energy carried away by and stored instantaneously in the fields are discussed only qualitatively in this paper, quantitative results for specific cloud parameters and scaling laws for the magnitude of the fields and the slowing down of the plasma cloud are presented in a companion manuscript.
\end{abstract}

\section{INTRODUCTION}

Examples of plasmas moving across magnetic fields can be found in laboratory experiments, active experiments in space, numerical simulations, and natural phenomena occurring in space plasmas and astrophysical plasmas. The expansion and convection of the energetic plasmas produced by high-altitude nuclear explosions (HANE's), ${ }^{1}$ the motion of plasma clouds released in the ionosphere and magnetosphere across the geomagnetic field, the ionized portion of rocket exhausts in the space environment, the orbital motion of plasmas produced by volcanic activity on the satellites of planets through the planetary magnetic fields (e.g., Io-Jupiter and Titan-Saturn systems), the eruption of magnetic loops and prominences in the solar corona, the motion of coronal ejecta through the solar magnetic field, the solar wind, the formation of auroral arcs, and the motion of galactic jets through the intergalactic magnetic field represent only a small subset of such phenomena. In addition, energetic plasmas that convect across an applied magnetic field are often produced in the laboratory by irradiating targets with intense laser pulses. These laser-produced plasmas can simulate conditions found in high-altitude nuclear explosions as well as less energetic phenomena associated with active experiments in space. The magnetic confinement of plasmas in fusion devices also often involves the cross-field motion of energetic plasmas.

The problem of modeling plasmas moving across magnetic field lines can be formulated in terms of five basic stages of evolution; namely, (1) plasma production, (2) plasma polarization and microscopic dynamics, (3) crossfield motion, (4) coupling to the background plasma and magnetic field, and (5) late-time plasma cloud dynamics. While the physical processes inherent to these various stages overlap in time, this partitioning provides an initial framework from which the intrinsic dynamical and morphological properties of cross-field plasma motion can be studied. Although many aspects of this problem have been addressed to varying degrees, no comprehensive model exists and indeed the transition from the early-time microscopic dynamics [encompassing stages (1)-(3)] to the late-time macroscopic evolution of the plasma [encompassing stages (3)-(5)] is poorly understood. In particular, while the radiative properties of the plasma have been characterized for time scales long compared to an ion gyroperiod (e.g., the Alfvén-wing model), the highfrequency, short-wavelength electromagnetic radiation emitted during the initial phases of polarization and the formation of charge layers has not been studied.

The late-time ( $t>100$ ion gyroperiods) plasma cloud dynamics and coupling to the background plasma and geomagnetic fields are subjects that have received considerable attention over the last two decades. ${ }^{2-4}$ The well-known Alfvén-wing model describes the cross-field motion and coupling to the background plasma and magnetic field and has been applied successfully to several phenomena in the solar system, including barium-cloud dynamics, ${ }^{3,5,6}$ IoJupiter coupling, ${ }^{7,8}$ auroral-arc formation, ${ }^{9,10}$ and magnetospheric-ionospheric coupling. ${ }^{4,11}$ In contrast, the early-time ( $t<100$ ion gyroperiods) dynamics has been investigated numerically only recently with both particle $^{12-18}$ and hybrid ${ }^{19}$ simulations (references therein). One of the inherent limitations of these electrostatic/electromagnetic numerical methods is their inability to manage large spatial and temporal domains. The neces- 
sity to resolve the individual particle motion over spatial scales less than a Debye length/ion gyroradius and times less than a plasma period/gyroperiod severely limits the spatial dimension and temporal extent that can be modeled. In addition, the density gradients are intrinsically large and can dominate the plasma dynamics observed in these simulations. A recent paper ${ }^{20}$ describes twodimensional hybrid simulations of ion beam propagation in a magnetoplasma. The evolution of beam density profiles is studied in the limit of high, dynamic plasma beta for beam dimensions much larger than an ion gyroradius, however, electron inertial effects are ignored and the early-time formation of the polarization field and the concomitant radiation field is not examined. A similar comment can be made regarding the large Larmor radius model developed by Hassam and $\mathrm{Huba}^{21}$ and applied by Huba et al. ${ }^{22}$ to the study of Rayleigh-Taylor-type instabilities that, in their opinion, describes the structure observed in the AMPTE magnetotail releases. Magnetohydrodynamic (MHD) calculations of cloud dynamics ${ }^{1,3,5,6}$ can alleviate some of the spatial and temporal restrictions imposed by particle/hybrid simulations, however, the low-frequency approximation does not allow for the evaluation of the earlytime electrostatic and radiation dynamics. An intermediate fully electromagnetic model is needed to bridge the gap between the early-time particle simulations and the latetime MHD calculations.

In this paper, a two-dimensional analytic solution of the coupled fluid and Maxwell's equations that allows for the description of the early-time dynamics associated with the formation of the polarization field and subsequent electromagnetic radiation is developed. A Fourier transform taken in one spatial dimension and a Laplace transform of these equations yield a set of integrodifferential equations for the electromagnetic fields. This set of equations is solved analytically and an inverse Laplace transform is then performed numerically. With this treatment it is possible to address the large spatial and temporal dimensions inherent to astrophysical and space plasmas, however, the nonlinear dynamics and large gradients associated with some laboratory experiments are not modeled. The carlytime development of the polarization fields and the subsequent electromagnetic radiation are observed and the transition from early to late times is obtained.

This paper is intended to be the first in a series and will address only the method of analysis and a zeroth-order analytic solution. A second companion paper (Paper II) describes the results for a particular parameter regime in some detail. In Paper II, we demonstrate quantitatively that, in the presence of radiation damping, the polarization field does not achieve a steady-state value and that the radiation field can carry away a substantial amount of the plasma cloud's momentum and kinetic energy on time scales of the order of the ion gyroperiod, depending on the plasma dimensions. The radiation spectrum obtained in Paper II consists of a burst of chirped, high-frequency (in the range of the cloud plasma frequencies) waves, followed by a pulse of whistler waves, and subsequently by ion cyclotron emission. Scaling laws are derived for the plasma momentum and energy loss rates and predictions for the braking time, the amplitude and spectrum of the radiation field, and the total radiated power are presented for conditions relevant to the recent CRRES chemical release experiments performed in the magnetosphere. Subsequent papers will incorporate additional complexity in the model including the effects of a background plasma and momentum and energy source terms. As suggested above, the method of analysis described in this paper can potentially be applied to many problems in space physics and astrophysics. In the case of active experiments, we are particularly interested in studying the overall braking of plasma clouds resulting from the momentum that is radiated away and the differential braking of the clouds that leads to a shearing off of charge layers and to the corresponding development of plasma density structures on a macroscopic scale. In addition, the spectral signature and magnitude of the radiated fields are potential diagnostics for remote sensing of plasmoids released in the Earth's atmosphere at high altitudes.

We begin in Sec. II of this paper with the development of our working set of equations. The fluid equations are linearized and the spatial and temporal ordering of the theory is discussed. Constitutive relations for the plasma currents are obtained from a Laplace transform of the fiuid equations. Maxwell's equations are Fourier transformed in one spatial dimension, a Laplace transform is performed, and the constitutive relations are incorporated to yield a set of coupled integrodifferential equations for the electromagnetic fields. The equations for the conservation of field and plasma momentum and energy are developed and are linearized. In Sec. III, the general solution is obtained and the normal modes of the system are discussed, while in Sec. IV, we summarize the results.

\section{THEORY}

The primary goal of this analysis is to quantify the early-time ( $<100$ ion gyroperiods) radiative properties of a plasma moving across a background magnetic field. In our model, the plasma is composed of electrons of mass $m_{e}$ and charge $q_{e}$ and ions of mass $m_{i}$ and charge $q_{i}$. As a first step, we choose to simplify the treatment of the plasma while retaining as much detail as possible in the mathematical model adopted for the generation and propagation of electromagnetic waves. Thus the electrons (ions) are assumed to have a Maxwellian velocity distribution function characterized, in general, by a density, $n_{e}\left(n_{i}\right)$, mean velocity, $\nabla_{e}\left(\nabla_{i}\right)$, and temperature $T_{e}\left(T_{i}\right)$. The geometry and initial conditions adopted for this problem are shown in Fig. 1. The plasma is assumed to be quasineutral and to have formed instantaneously with a velocity $V_{0}$ in the $y$ direction across a uniform background magnetic field with magnitude $B_{0}$ in the $z$ direction. The $\mathbf{V}_{0} \times \mathbf{B}_{0}$ component of the Lorentz force causes the plasma to polarize in the $x$ direction, resulting in a polarization field as shown in Fig. 1. The initial plasma density profile is taken to be Gaussian in the $x$ and $z$ directions with $1 / e$ half-widths of $D$ and $d$, respectively, and a peak density in the center of the cloud of magnitude $n_{0}$. In the $y$ direction, the plasma is assumed to be infinite in extent. As a result, no $E_{y}$ polarization field 
GEOMETRY

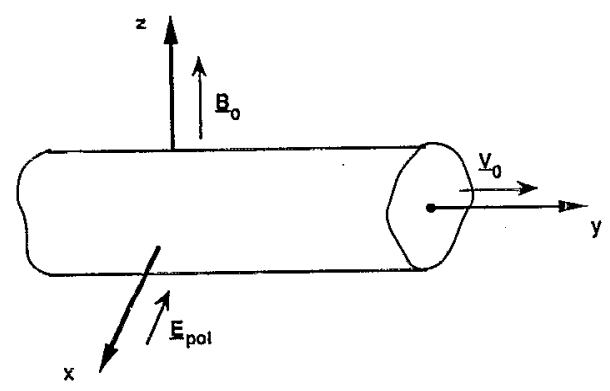

INITIAL CONDITIONS

$\begin{array}{ll}\text { Electron Density: } & n_{e}(t=0, x, z)=n_{0} e^{-x^{2}, D^{2} e-z^{2} / d^{2}} \\ \text { Ion Density: } & n_{i}(t=0, x, z)=n_{e}(t=0, x, z) \\ \text { Electron Velocity: } & v_{e}(t=0)=V_{0} \hat{y} \\ \text { Ion Velocity: } & \underline{v i}_{i}(t=0)=y_{e}(t=0) \\ \text { Electron Temperature: } & T_{e}=0 \\ \text { Ion Temperature: } & T_{i}=0 \\ \text { Electric Field: } & E(t=0)=0 \\ \text { Magnetic Field: } & B_{(t=0)=B_{0} \hat{z}}\end{array}$

FIG. 1. The geometry adopted for this problem is shown in the top panel. The plasma cloud is assumed to be infinite in the $y$ dircetion and to have formed instantaneously with a velocity $V_{0}$ across a uniform magnetic field, $B_{0}$ in the $z$ direction. A polarization field forms initially predominantly in the $x$ direction as shown. The initial conditions are listed in the bottom panel. Note that the initial plasma density profile is Gaussian in both $x$ and $z$.

can develop and only inductive fields exist in this direction.

The fluid equations needed to quantify the subsequent evolution of the plasma are presented in Sec. II A. These equations are coupled to Maxwell's equations, given in Sec. II $\mathrm{B}$, through the plasma currents. The constitutive relations obtained from the fluid equations are used to derive a single set of equations for the electromagnetic fields. Further approximations appropriate for the short temporal and large spatial scales of interest are discussed below. The exchange of momentum and energy between the plasma and the electromagnetic fields is treated in Sec. II C.

\section{A. Fluid equations of motion}

The fluid equations for a Maxwellian plasma are given by

$\frac{\partial}{\partial t} n_{j}+\nabla \cdot n_{j} \nabla_{j}=R$

$$
\begin{aligned}
\left(\frac{\partial}{\partial t}+\left(\mathbf{v}_{j} \cdot \nabla\right)\right) m_{j} n_{j} \mathbf{v}_{j}= & q_{j} n_{j}\left(\mathbf{E}+\frac{\mathbf{v}_{j} \times \mathbf{B}}{c}\right)-\nabla p_{j} \\
& -\sum_{k} n_{j} m_{j} v_{j k}\left(\mathbf{v}_{j}-\mathbf{v}_{k}\right)+m_{j} R \mathbf{v}_{j 0},
\end{aligned}
$$

$$
\begin{aligned}
\left(\frac{\partial}{\partial t}+\right. & \left.\left(\nabla_{j} \cdot \nabla\right)\right)\left(u_{j}+\frac{1}{2} m_{j} n_{j} v_{j}^{2}\right) \\
= & -\nabla \cdot\left(p_{j} \mathbf{v}_{j}\right)+q_{j} n_{j}\left(\mathbf{E}+\frac{\mathbf{v}_{j} \times \mathbf{B}}{c}\right) \cdot \nabla_{j} \\
& -\sum_{k} v_{j k}\left(\frac{1}{2} m_{j} n_{j} v_{j}^{2}-\frac{1}{2} m_{k} n_{k} v_{k}^{2}+u_{j}-u_{k}\right)+R u_{0},
\end{aligned}
$$

where the subscript $j$ refers to the $j$ th particle species (electrons or ions), the subscript $k$ includes a background plasma and neutral particles, $R$ is the rate per unit volume at which plasma is produced (e.g., by photoionization of neutrals), $n_{j}, \mathbf{v}_{j}$ and $u_{j}$ are the $j$ th particle density, mean velocity, and thermal energy density, respectively, $p_{j}$ is the $j$ th particle partial pressure, $v_{j k}$ is the collision frequency between $j$ th and $k$ th particle species, $q_{j}\left(\mathbf{E}+v_{j} \times \mathbf{B} / c\right)$ is the Lorentz force associated with self-consistent electric (E) and magnetic (B) fields, and we have allowed for the production of particle momentum $\left(m_{j} R \mathbf{v}_{j 0}\right)$ and thermal energy $\left(R u_{0}\right)$. For a Maxwellian velocity distribution function, the $j$ th particle partial pressure and thermal energy density are defined as $n_{j} k_{\mathrm{B}} T_{j}$ and $3 n_{j} k_{\mathrm{B}} T_{j} / 2$, with $T_{j}$ equal to a temperature and $k_{\mathrm{B}}$ equal to Boltzmann's constant.

In order to identify the dominant terms in these equations for the temporal and spatial scales of interest, we introduce the following scaling parameters:

$L=$ spatial dimension of the plasma $(=d$ or $D)$,

$V_{0}=$ initial plasma velocity across the background magnetic field,

$B_{0}=$ background magnetic field strength,

$E_{0}=V_{0} B_{0} / c$, the polarization electric field necessary to allow the plasma to drift across the ambient magnetic field,

$\tau=m_{i} c / q_{i} B_{0}$, an ion gyroperiod,

$n_{0}=$ the initial plasma density in the center of the cloud,

$W_{j 0}=n_{0} m_{j} V_{0}^{2} / 2$, the initial kinetic energy density of the $j$ th particle species.

Normalizing the spatial coordinate, plasma velocities, magnetic field, electric field, temporal coordinate, plasma densities, and $j$ th particle pressure and thermal energy to the above quantities, respectively, yields the following nondimensional equations:

$$
\begin{aligned}
\frac{\partial}{\partial t} n_{j}+\frac{\rho_{i}}{L} \nabla \cdot n_{j} \mathbf{v}_{j}=\tau R, & \\
\left(\frac{\partial}{\partial t}+\frac{\rho_{i}}{L}\left(\mathbf{v}_{j} \cdot \nabla\right)\right) \mathbf{v}_{j}= & \tau \Omega_{j}\left(\mathbf{E}+\frac{\mathbf{v}_{j} \times \mathbf{B}}{c}\right)-\frac{\rho_{i}}{L} \frac{1}{2 n_{j}} \nabla p_{j} \\
& -\tau \sum_{k} v_{j k}\left(\mathbf{v}_{j}-\mathbf{v}_{k}\right) \\
& +\tau R\left(\frac{\mathbf{v}_{j 0}}{v_{0}}-\mathbf{v}_{j}\right),
\end{aligned}
$$




$$
\begin{aligned}
\frac{\partial}{\partial t} T_{j}+\frac{\rho_{i}}{L}\left(\mathbf{v}_{j} \cdot \nabla\right) T_{j}= & -\frac{2}{3} \frac{\rho_{i}}{L} T_{j} \nabla \cdot \mathbf{v}_{j}-\tau \sum_{k} v_{j k} \\
& \times\left(T_{j}-T_{k}\right)+\tau R\left(T_{0}-T_{j}\right),
\end{aligned}
$$

where we have adopted the notation in Eq. (1) through Eq. (3) for the nondimensional variables, $\rho_{i}$ is an ion gyroradius $\left(=V_{0} \tau\right)$ and $\Omega_{j}$ is the $j$ th particle gyrofrequency. Note that the spatial derivatives have a scaling given by the ratio of the ion gyroradius to the overall dimension of the plasma and that the collisional interaction and production terms scale as the rate divided by the ion gyrofrequency. As a first approximation, we will assume that $\rho_{i} / L<1$, $\tau R<1$, and $v_{j k} \tau<1$, yiclding the simplified set of fluid equations given by,

$$
\begin{aligned}
& \frac{\partial}{\partial t} n_{j}=0, \\
& \frac{\partial}{\partial t} \mathbf{v}_{j}=\tau \Omega_{j}\left(\mathbf{E}+\frac{\mathbf{v}_{j} \times \mathbf{B}}{c}\right), \\
& \frac{\partial}{\partial t} T_{j}=0 .
\end{aligned}
$$

Thus, for large-scale plasmas, the dynamics of cross-field plasma motion is dominated by the interaction of the radiation field with the plasma via the Lorentz force. If we further assume that the induced magnetic fields are small in magnitude compared to the background magnetic field, it is possible to linearize the momentum equation [Eq. (8)] with the result

$$
\frac{\partial}{\partial t} \mathbf{v}_{j}=\frac{q_{j}}{m_{j}}\left(\mathbf{E}+\frac{\mathbf{v}_{j} \times \mathbf{B}_{0}}{c}\right),
$$

where we have reverted back to the dimensional form of the equations. This approximation is valid in the nonrelativistic limit and, provided that the initial plasma kinetic energy density is small, compared to the background magnetic field energy density [i.e., $\left.\beta=4 \pi\left(n_{e} m_{e}+n_{i} m_{i}\right) V_{0}^{2} / B_{0}^{2} \ll 1\right]$. If the latter condition werc not met, than the plasma would be able to create large corrections to the ambient field as is obtained in the formation of diamagnetic cavities.

The constitutive relations for the plasma currents can be derived by taking the Laplace transform of Eq. (10) for the electrons and ions and combining the resulting equations with the expression for the current $\left(\mathrm{J}=n_{i} q_{i} \mathbf{v}_{i}+n_{e} q_{e} \mathbf{v}_{e}\right)$ with the result

$$
\widetilde{\mathbf{J}}=\boldsymbol{\sigma} \cdot \widetilde{\mathbf{E}}+\frac{\sigma_{\mathrm{P}}}{s} \frac{V_{0} B_{0}}{c} \hat{x}-\frac{\sigma_{\mathrm{H}}}{s} \frac{V_{0} B_{0}}{c} \hat{y},
$$

where $s$ is the Laplace transform variable, $\sigma$ is a tensor given by

$$
\boldsymbol{\sigma}=\left[\begin{array}{ccc}
\sigma_{\mathrm{p}} & \sigma_{\mathrm{H}} & 0 \\
-\sigma_{\mathrm{H}} & \sigma_{\mathrm{p}} & 0 \\
0 & 0 & \sigma_{\|}
\end{array}\right]
$$

and $\sigma_{\mathrm{P}}, \sigma_{\mathrm{H}}$, and $\sigma_{\|}$are defined as

$$
\begin{aligned}
& \sigma_{\mathrm{P}}=\frac{n_{e}(t=0) e^{2}}{m_{e}} \frac{s}{\omega_{c c}}\left(\frac{\omega_{c i}}{\left(s^{2}+\omega_{c i}^{2}\right)}+\frac{\omega_{c e}}{\left(s^{2}+\omega_{c e}^{2}\right)}\right), \\
& \sigma_{\mathrm{H}}=\frac{n_{e}(t=0) e^{2}}{m_{e}} \frac{1}{\omega_{c e}}\left(\frac{\omega_{c i}^{2}}{\left(s^{2}+\omega_{c i}^{2}\right)}+\frac{\omega_{c e}^{2}}{\left(s^{2}+\omega_{c e}^{2}\right)}\right), \\
& \sigma_{\|}=\frac{n_{e}(t=0) e^{2}}{m_{e}} \frac{\left(1+m_{e} / m_{i}\right)}{s},
\end{aligned}
$$

where $\omega_{c i}, \omega_{c e}$ equal to the ion and electron gyrofrequencies, respectively. Equation (12) is the inertial equivalent of the conductivity tensor found in collision-dominated transport theory. The quantities $\sigma_{\mathrm{p}}, \sigma_{\mathrm{H}}$, and $\sigma_{\|}$correspond to the Pedersen, Hall, and parallel conductivities derived for a plasma in a magnetic field with the inertial parameter $s$ (the Laplace transform variable) replacing the usual collision rate. Equation (11) for the currents is in dimensional form and contains terms proportional to $V_{0} B_{0} / c$. As the plasma attempts to move across the magnetic field, inertial separation of the electrons and ions occurs and produces an oscillating polarization electric field. The corresponding oscillating currents represented by the two terms proportional to $V_{0} B_{0} / c$ in Eq. (11) enter Maxwell's equations as source terms that drive the production of electromagnetic radiation.

\section{B. Maxwell's equations}

The self-consistent evolution of the electromagnetic fields is obtained from Maxwell's equations, which can be combined to yield a single-vector equation for the electric fields; namely,

$$
\frac{\partial^{2} \mathbf{E}}{\partial t^{2}}-c^{2} \nabla^{2} \mathbf{E}+c^{2} \nabla(\nabla \cdot \mathbf{E})=-4 \pi \frac{\partial \mathbf{J}}{\partial t}
$$

Given that the plasma is infinite in the $y$ direction $(d / d y$ $=0$ ) and taking the Laplace transform and Fourier transform in $x$, Eq. (16) can be written

$$
s^{2} \widetilde{E}_{x}^{k}-c^{2} \frac{d^{2} \widetilde{E}_{x}^{k}}{d z^{2}}-i k c^{2} \frac{d \widetilde{E}_{z}^{k}}{d z}=-4 \pi s \widetilde{J}_{x}^{k}
$$




$$
\begin{aligned}
& \left(s^{2}+c^{2} k^{2}\right) \widetilde{E}_{y}^{k}-c^{2} \frac{d^{2} \widetilde{E}_{y}^{k}}{d z^{3}}=-4 \pi s \widetilde{J}_{y}^{k}, \\
& \left(s^{2}+c^{2} k^{2}\right) \tilde{E}_{z}^{k}-i k c^{2} \frac{d \widetilde{E}_{x}^{k}}{d z}=-4 \pi s \widetilde{J}_{z}^{k}
\end{aligned}
$$

Performing the same transforms on the vector components of Faraday's equation yields

$$
\begin{aligned}
& s \widetilde{B}_{x}^{k}=c \frac{d \widetilde{E}_{y}^{k}}{d z}, \\
& s \widetilde{B}_{y}^{k}=c\left(i k \widetilde{E}_{z}^{k}-\frac{d \widetilde{E}_{x}^{k}}{d z}\right), \\
& s \widetilde{B}_{z}^{k}=-i k c \widetilde{E}_{y}^{k},
\end{aligned}
$$

where $k$ is the $x$-Fourier transform variable, where the superscript $k$ designates a Fourier transform, and where we have taken the initial value and initial temporal derivative equal to zero for all field quantities over all space. The transformed currents involve a convolution between the initial density profiles and the electric fields, given by

$$
\begin{aligned}
\widetilde{\mathbf{J}}^{k}= & \left(\Sigma \cdot \int_{-\infty}^{\infty} d k^{\prime} n^{k-k^{\prime}} \widetilde{\mathbf{E}}^{k^{\prime}}+\Sigma_{\mathrm{P}} \frac{n^{k}}{s} \frac{V_{0} B_{0}}{c} \hat{x}\right. \\
& \left.-\Sigma_{\mathrm{H}} \frac{n^{k}}{s} \frac{V_{0} B_{0}}{c} \hat{y}\right),
\end{aligned}
$$

where $\Sigma$ represents normalized conductivities $[\Sigma=\sigma / n(t$ $=0, x, z)]$ and $n^{k}(z)$ is the Fourier transform of the initial plasma density; namely,

$$
n^{k}(z)=\sqrt{\pi} D e^{-z^{2} / d^{2}} \cdot n_{0} e^{-k^{2} D^{2} / 4}
$$

Equations (17)-(19) together with Eq. (23) form a couplet set of integrodifferential equations in $k$ and $z$ for the three electric field components. The magnetic field components are found from Eqs. (20)-(22) given a solution for the electric fields while the plasma velocities can be derived from the constitutive relations. The plasma density is constant in time to zeroth order in the parameter $\rho_{i} / L$. However, it is possible to compute the magnitude of the density perturbations associated with the formation of charge layers from a linearization of Eq. (4), including the spatial derivative term; namely,

$$
\frac{\partial}{\partial t} n_{j}=-\frac{\rho_{i}}{L} \nabla \cdot n_{j}(t=0) \nabla_{j}
$$

Thus the plasma density perturbation is of order $\left(\rho_{i} / L\right)$ times the initial plasma density. The method of analysis for solving these equations is discussed below.

\section{Total momentum and energy conservation}

Having assumed a collisionless plasma, that the interaction of the jetting plasma with any existing background plasma is negligible, and that the rate of momentum and energy production can be neglected over the short time scales of interest, it is evident that the total momentum and energy stored in the electromagnetic fields and the jetting plasma over all space must remain constant for all time. Thus momentum and energy are exchanged with time between the plasma and the fields, as defined quantitatively by the equations

$$
\begin{aligned}
& \frac{\partial}{\partial t}\left(\mathbb{P}+\mathbf{P}_{f}\right)=-\nabla \cdot \mathbf{P v}+\nabla \cdot \mathrm{M}, \\
& \frac{\partial}{\partial t}\left(w+w_{f}\right)=-\nabla \cdot w \mathbf{v}-\nabla \cdot \mathbf{S},
\end{aligned}
$$

where $\mathbb{P}\left(=n_{i} m_{i} \mathbf{v}_{i}+n_{e} m_{e} \mathbf{v}_{e}\right)$ is the momentum density in the plasma, $\mathbf{P}_{f}(=\mathbf{E} \times \mathbf{B} / 4 \pi c)$ is the momentum density stored in the fields, $\mathbf{v}\left[=\left(n_{i} m_{i} \mathbf{v}_{l}+n_{e} m_{e} \mathbf{v}_{e}\right) /\left(n_{i}+n_{e}\right)\right]$ is the mean velocity of the plasma, $M_{\alpha \beta}\left\{=\left[E_{\alpha} E_{\beta}+B_{\alpha} B_{\beta}\right.\right.$ $\left.\left.-\left(E^{2} B^{2}\right) \delta_{\alpha \beta} / 2\right] / 4 \pi\right\}$ is the Maxwell stress tensor, $w$ $\left(=n_{i} m_{i} v_{i}^{2} / 2+n_{e} m_{e} v_{e}^{2} / 2\right)$ is the plasma kinetic energy density, $w_{f}\left[=\left(E^{2}+B^{2}\right) / 8 \pi\right]$ is the wave energy density, and $\mathbf{S}[\equiv c(\mathbb{E} \times \mathbf{B}) / 4 \pi]$ is the Poynting flux. In nondimensional form, Eqs. (26) and (27) become

$$
\begin{aligned}
& \frac{\partial}{\partial t}\left(\mathbf{P}+\mathbf{P}_{f}\right)=-\frac{\rho_{i}}{L} \nabla \cdot \mathbf{P} \mathbf{v}+\frac{\rho_{i}}{L} \nabla \cdot \mathbf{M}, \\
& \frac{\partial}{\partial t}\left(w+w_{f}\right)=-\frac{\rho_{i}}{L} \nabla \cdot w \mathbf{v}-\frac{\rho_{i}}{L} \nabla \cdot \mathbf{S}
\end{aligned}
$$

where the plasma and field quantities are normalized as before. To zeroth order in $\rho_{i} / L$, it is possible to neglect the divergence of the plasma momentum and energy fluxes. However, the Maxwell stress tensor and the Poynting flux both contain terms of order $c^{2} / V_{0}^{2}$ times $B / B_{0}$ so that the divergence of the field momentum and energy fluxes cannot be neglected. Thus, to zeroth order in $\rho_{i} / L$, Eqs. (28) and (29) become

$$
\begin{aligned}
& \frac{\partial}{\partial t}\left(\mathbf{P}+\mathbf{P}_{f}\right)=\frac{\rho_{i}}{L} \nabla \cdot \mathbf{M}, \\
& \frac{\partial}{\partial t}\left(w+w_{f}\right)=-\frac{\rho_{i}}{L} \nabla \cdot \mathbf{S} .
\end{aligned}
$$

It is interesting to note that, in the presence of a strong background magnetic field, electromagnetic radiation carries away a much larger fraction of the plasma momentum density than energy density. This effect is depicted in Fig. 2. While the electromagnetic radiation launched along the background field by the plasma has an energy density proportional to $E_{x} \times B_{y}$, the $y$-momentum density is proportional to $E_{x} \times B_{0}$. Given that $B_{0}$ is large compared to $B_{y}$ the relative proportion of momentum density carried by the electromagnetic wave can be significantly larger than 

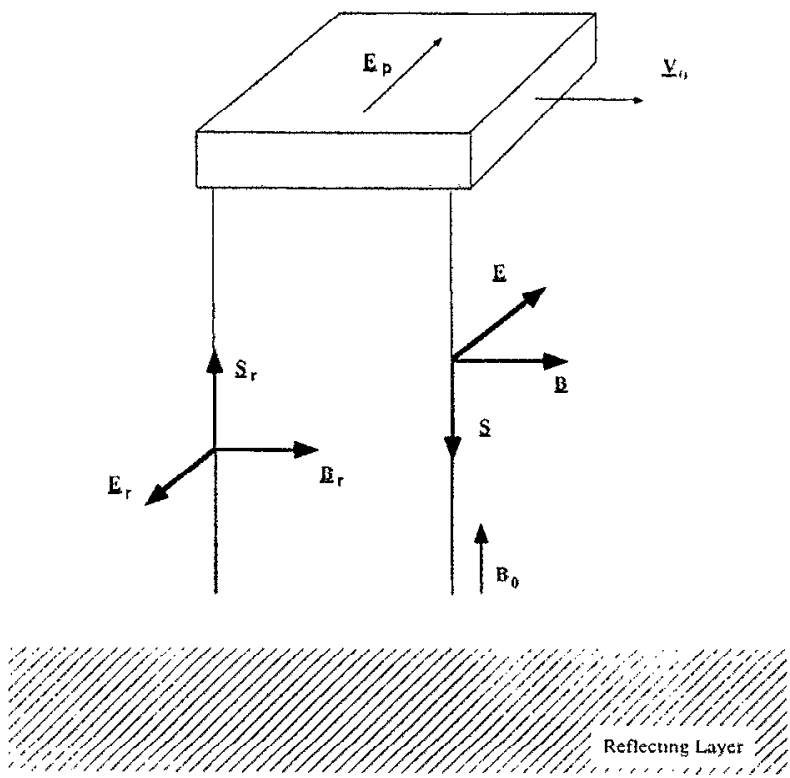

FIG. 2. The radiation field $(\mathbf{E}, \mathbf{B})$ launched by a plasma slab moving across a uniform background magnetic field $\mathbf{B}_{0}$ is depicted schematically. The wave propagates along the field with Poynting vector $\mathbf{S}$ as shown and carries $y$-momentum density proportional to $E_{x} \times B_{0}$. The energy density carried by the wave is proportional to $E_{x} \times B_{y}$. The reflected wave $\left(\mathbf{E}_{\mathrm{r}}, \mathbf{B}_{\mathrm{r}}\right)$ produced in the presence of a conducting boundary is shown propagating back toward the plasma cloud. This wave carries negative momentum which acts to slow the plasma cloud.

the corresponding energy density. Figure 2 also illustrates what happens when a reflecting boundary is present. In the case of a perfectly conducting boundary, the reflected fields are equal in magnitude to the incident fields and twice the incident $y$ momentum is given up to the reflecting medium while the reflected wave carries negative $y$ momentum back to the plasma cloud causing it to slow. This effect can be important for both laboratory and space experiments and has been discussed previously in the context of lowfrequency waves (Alfvén-wing model), ${ }^{5,6}$

\section{SOLUTION}

\section{A. Ansatz}

Two factors enter into the development of an analytical solution to Eqs. (17)-(19). The first is associated with the large physical dimensions of the plasmas of interest relative to the short-wavelength electromagnetic waves that are launched during the early phases of evolution of the plasma. This consideration together with the fact that oscillating currents are set up primarily in a direction perpendicular to the magnetic field leads us to seek a solution that allows for wave propagation predominantly along the magnetic field. In this limit, the plasma appears to be a large radiating dipole antenna oriented perpendicular to both the magnetic field and the direction of motion. The second factor pertains to the Fourier transform [Eq. (23)] of the currents. The Gaussian weighting term in the convolution integrals suggests an expansion for the electric field components in terms of Hermite polynomials that form a complete orthogonal set over the interval $k=-\infty$ to $\infty$ and satisfy the orthogonality relation

$$
\int_{-\infty}^{\infty} d k H_{n}(k) H_{m}(k) e^{-k^{2}}=2^{n} \pi^{1 / 2} n ! \delta_{n m}
$$

where $H_{n}(k)$ is a Hermite polynomial of order $n$. Hermite polynomials, described in Abramowitz and $\operatorname{Stegun}^{23}$ ( $\mathrm{p}$. 773), have increasing magnitude at large $k$ with increasing order. Their limiting value for large $k$ is given by

$$
\left|H_{n}(k)\right|<1.086 e^{k^{2} / 2} 2^{n / 2}
$$

while the generating function for these polynomials is written

$$
H_{n}(k)=(-1)^{n} e^{k^{2}} \frac{d^{n}}{d k^{n}} e^{-k^{2}}
$$

with, for example, the results $H_{0}(k)=1$ and $H_{1}(k)=2 k$.

These factors lead us to propose an ansatz for the electric fields that includes an expansion in terms of a finite number $N$ of Hermite polynomials and that allows for wave propagation predominantly in the $z$ direction. Further, we anticipate that our system of equations will define a finite number of wave modes that propagate in the $z$ direction and that the eigenvalues for these modes will be related to the wave numbers in the $z$ direction of the waves. The associated eigenvectors, in turn, will define the relative magnitude of the Hermite polynomial terms for the given wave mode and for each coordinate component of the field. With these considerations and given that the total number of wave modes for a given order of approximation $N$ is $2 N+2$ (determined a posteriori), the ansatz for the fields can be written

$$
\begin{gathered}
\widetilde{E}^{\beta}=\sum_{n=0}^{N} e^{-k^{2} D^{2} / 4} H_{n}\left(\frac{k D}{2}\right) \widetilde{E}_{H}^{\beta}(s, z), \\
\widetilde{\mathbf{E}}_{H}(s, z)=\sum_{m=0}^{2 N+1} \mathbf{e}_{m}(s, z)\left[a_{m}(s, z) e^{i \int_{0}^{z} k_{z^{\prime \prime}}^{m} d z^{\prime \prime}}\right. \\
\left.+b_{m}(s, z) e^{-i \int_{0}^{z} k_{z^{\prime \prime}}^{m} d z^{\prime \prime}}\right],
\end{gathered}
$$

where $k_{z}^{m}$ is the wave number (eigenvalue) of the $m$ th normal mode and $\mathbf{e}_{m}$ is the corresponding eigenvector, which, together with $\mathbf{E}_{H}$, possesses the general form 


$$
\mathbf{e}_{m}=\left[\begin{array}{c}
e_{0}^{x} \\
\cdot \\
\cdot \\
e_{N}^{x} \\
e_{0}^{y} \\
\cdot \\
e_{N}^{y} \\
e_{0}^{z} \\
\cdot \\
\cdot \\
e_{N}^{z}
\end{array}\right]_{m}
$$

and $a_{m}$ and $b_{m}$ are unknown coefficients that depend, in general, on $s$ and $z$ and that define the relative contribution of the $m$ th mode to the amplitude of the fields.

Because the Hermite polynomial is such that higherorder terms have increasing amplitude with increasing $k$, we anticipate that the expansion (35) will include additional effects associated with smaller and smaller scale structures in the $x$ direction as the order of the expansion increases. The accuracy of the results for a given order will therefore depend on the degree to which small-scale structures are important and modeled to that order as the plasma cloud and radiation field evolve in time.

\section{B. Green's function solution}

We proceed by substituting the ansatz Eq. (35) into Eqs. (17)-(19) and assume that the dimension of the plasma clouds of interest are much larger than the vacuum wavelength or the dielectric wavelength of the radiation emitted at early times. This assumption is equivalent to that of geometrical optics ${ }^{24}$ and allows us to ignore terms that involve derivatives of the coefficients with respect to $z$ relative to the time-derivative terms (e.g., $d^{2} a_{m} / d z^{2} / a_{m} \ll s^{2} / c^{2}$ ) and/or dielectric terms (e.g., $d^{2} a_{m} / d z^{2} / a_{m}<k_{z}^{2}$ ). In addition, we dot (the dot product operation is defined in the Appendix) the resulting equations with a Hermite polynomial of the order $l$, invoking the orthogonality condition Eq. (32) whenever possible. The net result is an equation of the form

$$
\left(\mathrm{A} k_{z}^{2}+\mathrm{B} k_{z}+\mathrm{C}\right) \cdot \mathbf{a}=\mathbf{S},
$$

where $A, B$, and $C$ are matrices whose elements, derived in the Appendix are functions of $s$ and $z$, a is a vector composed of unknown Hermite polynomial coefficients, and $\mathbf{S}$ is a vector containing the source term that has also been dotted with $H_{l}(k D / 2)$. The matrices take on the form,

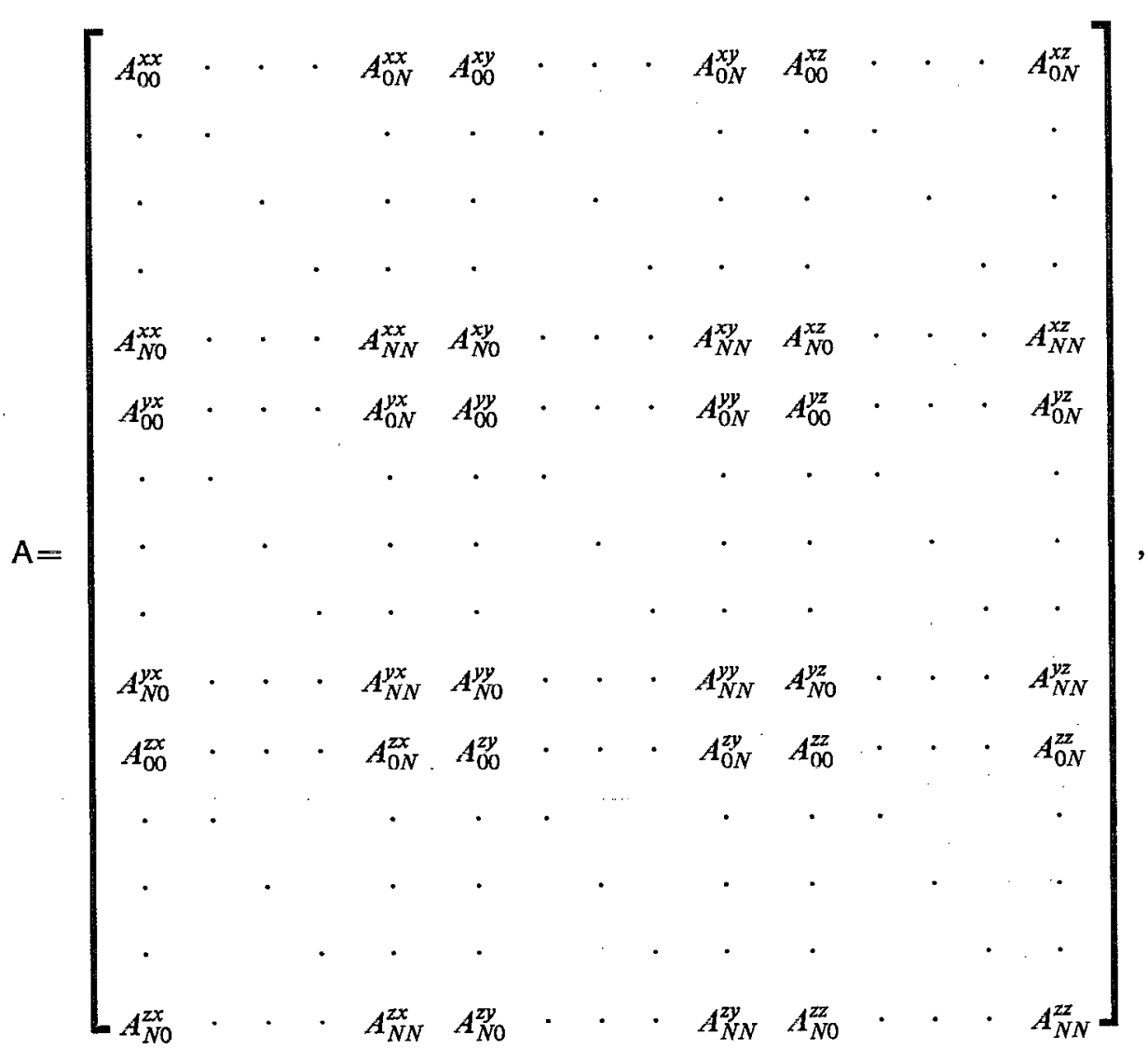


where we have adopted a notation for the matrix elements such that the left superscript refers to the $x, y$, or $z$ equations [i.e., Eqs. (17), (18), or (19), respectively], the right superscript refers to the $x, y$, or $z$ component of the field on which the matrix is operating, the left subscript corresponds to the lth Hermite polynomial with which the equations were dotted, and the right superscript corresponds to the particular Hermite polynomial component of the field. Any element of the matrix can be represented in index notation as $A_{l n}^{\alpha \beta}$, where the superscripts given as Greek letters indicate the particular wave equation or coordinate as described above and the subscripts are taken to be Arabic letters and denote the order of the Hermite polynomial components of the fields or dotted into the equations. The vectors $\mathbf{a}$ and $\mathbf{S}$ both take on a form similar to the eigenvectors defined above; namely,

$$
\mathbf{a}=\left[\begin{array}{c}
a_{0}^{x} \\
\cdot \\
\cdot \\
a_{N}^{x} \\
a_{0}^{y} \\
\cdot \\
a_{N}^{y} \\
a_{0}^{z} \\
\cdot \\
\cdot \\
\cdot \\
a_{N}^{z}
\end{array}\right],
$$

where the vector components possess a notation similar to that of the matrix elements, the superscript referring to the particular field coordinate and the subscript representing the Hermite polynomial component. The vector in index notation is written $a_{n}^{\beta}$. Equation (38) represents an intermediate step to finding a Green's function solution to Eqs. (17)-(19). The homogeneous equations are found by setting the forcing terms in Eq. (38) to zero. Setting the determinant of the left-hand matrix to zero then yields the normal modes of the system (the $k_{z}^{m}$ ) while the homogeneous equation with the appropriate eigenvalues substituted in yields the corresponding eigenvectors $\left(e_{m}\right)$.

To determine the Green's function, we must find the response at position $z$ and spatial frequency $k$ of our system of equations to a disturbance initiated at $z^{\prime}$ and $k^{\prime}$ for each of the components of the forcing term, including the particular equation that is being driven [Eqs. (17)-(19)] and the particular order of the Hermite polynomial that is driving the equation. Thus we search for Green's function solutions to equations of the form

$$
\begin{aligned}
& L_{l} \frac{d^{2}}{d z^{2}} \cdot \widetilde{\mathbf{G}}^{\gamma}+\mathrm{L}_{2} \frac{d^{2}}{d z^{2}} \cdot \widetilde{\mathbf{G}}^{\gamma}+\mathrm{L}_{3} \cdot \widetilde{\mathbf{G}}^{\gamma}+\mathrm{L}_{4} \cdot \widetilde{\mathbf{G}}^{\gamma} \\
& \quad=\delta\left(z-z^{\prime}\right) \delta\left(k-k^{\prime}\right) \mathbf{I}^{\gamma},
\end{aligned}
$$

with

$$
\mathbf{I}^{x}=\left[\begin{array}{l}
1 \\
0 \\
0
\end{array}\right], \quad \mathbf{I}^{y}=\left[\begin{array}{l}
0 \\
1 \\
0
\end{array}\right], \quad \mathbf{I}^{z}=\left[\begin{array}{l}
0 \\
0 \\
1
\end{array}\right],
$$

and where the matrix operators $L$ are given by

$$
\begin{aligned}
& L_{1}=\left[\begin{array}{ccc}
-c^{2} & 0 & 0 \\
0 & -c^{2} & 0 \\
0 & 0 & 0
\end{array}\right], \\
& L_{2}=\left[\begin{array}{ccc}
0 & 0 & -i k c^{2} \\
0 & 0 & 0 \\
-i k c^{2} & 0 & 0
\end{array}\right], \\
& L_{3}=\left[\begin{array}{ccc}
s^{2} & 0 & 0 \\
0 & s^{2}+c^{2} k & 0 \\
0 & 0 & s^{2}+c^{2} k^{2}
\end{array}\right],
\end{aligned}
$$

where $O\left(k, k^{\prime}\right)$ equal to an integral operator given by

$$
O\left(k, k^{\prime}\right)=D \pi^{1 / 2} e^{-z^{2} / d^{2}} \int \frac{d k^{\prime}}{2 \pi} e^{-\left(k^{-} k^{\prime}\right)^{2} D^{2} / 4}
$$

The Green's function matrices have the form

$$
\widetilde{\mathbf{G}}^{\gamma}=\left[\begin{array}{c}
\widetilde{G}^{x \gamma} \\
\widetilde{G}^{y \gamma} \\
\widetilde{G}^{z \gamma}
\end{array}\right]
$$


where the superscript $\gamma$ takes on the values $x, y$, or $z$ corresponding to the appropriate equation and $\widetilde{\mathbf{G}}^{\gamma}$ represents the natural response of our system of equations to an impulse at $k=k^{\prime}$ and $z=z^{\prime}$ appearing in the $\gamma$ equation.

Now we allow for the expansion of our solution in terms of Hermite polynomials and write the Green's function given above in a way that is similar to the ansatz developed for the electric field; namely,

$$
\widetilde{G}^{\beta \gamma}(s, k, z)=\sum_{n=0}^{N} e^{-k^{2} D^{2} / 4} H_{n}\left(\frac{k D}{2}\right) \widetilde{G}_{H n}^{\beta \gamma}(s, z),
$$

with

$$
\begin{aligned}
\widetilde{\mathbf{G}}_{H}^{\gamma}(s, z)= & \sum_{m=0}^{2 N+1} \mathbf{e}_{m}(s, z)\left[g_{m}^{\gamma}(s, z) e^{i \int_{0}^{z} k_{z^{\prime \prime}}^{m} d z^{\prime \prime}}\right. \\
& \left.+h_{m}^{\gamma}(s, z) e^{-i \int_{0}^{z} k_{z^{\prime \prime}}^{m} d z^{\prime \prime}}\right],
\end{aligned}
$$

where the coefficients $g_{m}^{\gamma}$ and $h_{m}^{\gamma}$ must be determined ultimately from continuity and jump conditions. Equation (49) is then substituted into Eq. (41) and the resulting equation dotted with a Hermite polynomial of order $l$ to yield

$$
\begin{aligned}
& \sum_{n=0}^{N} \sum_{\beta} \sum_{m=0}^{2 N+1}\left(A_{l n}^{\alpha \beta} \frac{d^{2}}{d z^{2}}+B_{l n}^{\alpha \beta} \frac{d}{d z}+C_{l n}^{\alpha \beta}\right) \\
& \quad \times\left\{e_{n m}^{\beta}\left[g_{l m}^{\gamma}(s, z) e^{i \int_{0}^{z} k_{z^{\prime \prime}}^{m} d z^{\prime \prime}}+h_{i m}^{\gamma}(s, z) e^{-i \int_{0}^{z} k_{z^{\prime \prime}}^{m} d z^{\prime \prime}}\right]\right\} \\
& \quad=H_{1}\left(\frac{k^{\prime} D}{2}\right) \delta_{\alpha \gamma} \delta_{l i} \delta\left(z-z^{\prime}\right),
\end{aligned}
$$

where we have used index notation for the matrices to simplify the result, $A_{l n}^{\alpha \beta}, B_{l n}^{\alpha \beta}$, and $C_{l n}^{\alpha \beta}$, are elements of the matrices obtained in the Appendix, and the index $i$ represents the particular Hermite polynomial component of the applied impulse. Because we have incorporated the eigenvalues and eigenvectors into the expression [Eq. (15)], the Green's function automatically satisfies Eq. (51) for $z<z^{\prime}$ and $z>z^{\prime}$; however, the coefficients are different in each case and will be designated $g_{i m}^{\gamma>}, h l_{m}^{\gamma>}$ for $z>z^{\prime}$ and $g_{i m}^{\gamma^{<}}$, $h_{i m}^{\gamma<}$ for $z<z^{\prime}$. To solve for these coefficients, we require continuity of the Green's function across $z=z^{\prime}$ and that the jump conditions obtained by integrating. Eq. (51) be satisfied; namely,

$$
\begin{aligned}
& \sum_{n=0}^{N} \sum_{\beta} \sum_{m=0}^{2 N+1} A_{l n}^{\alpha \beta} i k_{z^{\prime}}^{m} e_{n m}^{\beta}\left[g_{l m}^{\gamma>}(s, z) e^{i \int_{0}^{z} k_{z^{\prime \prime}}^{m} d z^{\prime \prime}}\right. \\
& \left.+h_{i m}^{\gamma<}(s, z) e^{\left.-i \int_{0}^{z} k_{z^{\prime \prime}}^{m} d z^{\prime \prime}\right]}\right]=H_{1}\left(\frac{k^{\prime} D}{2}\right) \delta_{\alpha \gamma} \delta_{l i}
\end{aligned}
$$

In addition, causality requires that only waves propagating in the positive (negative) $z$ direction be allowed for $z>$ (<) $z^{\prime}$; so that $h_{l m}^{\gamma>}=0$ and $g_{i m}^{\gamma^{<}}=0$. Realizing that, for the homogeneous equations, the $z$-component coefficients of the Green's function are given in terms of the $x$ coefficients by the $\alpha=z$ equation in Eq. (51), we count $8(N+1)^{2}$ unknown Green's function coefficients. Similarly, the continuity condition yields $4(N+1)^{2}$ unique equations while the jump conditions also yield $4(N+1)^{2}$ equations. Thus we are left with $8(N+1)^{2}$ coefficients and $8(N+1)^{2}$ equations from the continuity and jump conditions combined.

Given the normal modes and corresponding eigenvectors together with the continuity requirement and the jump conditions, it is possible to obtain the final form for the Green's function. The general solution for the fields is then calculated by dotting the Green's function with the source term and integrating over $k^{\prime}$ and $z^{\prime}$. Thus the fields are given by Eq. (35) with

$$
\begin{aligned}
\widetilde{E}_{H n}^{\beta}(s, z)= & \sum_{i=0}^{N} \sum_{\gamma} \sum_{m=0}^{2 N+1} e_{n m}^{\beta}(s, z) \int_{-\infty}^{\infty} d k^{\prime} H_{i}\left(\frac{k^{\prime} D}{2}\right) \\
& \times\left(\int_{-\infty}^{z} d z^{\prime} g_{i m}^{\gamma>}\left(s, k^{\prime}, z^{\prime}\right) S_{i}^{\gamma}\left(s, z^{\prime}\right)\right. \\
& \left.+\int_{z}^{\infty} d z^{\prime} h_{i m}^{\gamma<}\left(s, k^{\prime}, z^{\prime}\right) S_{i}^{\gamma}\left(s, z^{\prime}\right)\right)
\end{aligned}
$$

where the $S \gamma$ are the source term coefficients obtained in the Appendix. Note that we have derived the source terms after dividing our equations by $V_{0} B_{0} / c$ so that the electric field is now normalized to this factor.

Clearly, it is not possible to write a closed form solution for the Green's function without limiting the number of terms in the expansion in terms of Hermite polynomials. In the remainder of this paper, we derive the zeroth-order solution, which not only demonstrates the methodology for obtaining a general $N$ th-order result but also illustrates many of the very interesting aspects of wave generation and propagation in a two-dimensional plasma.

\section{Zeroth-order solution}

The first step in obtaining the zeroth-order solution is to determine the normal modes and the corresponding eigenvectors. Given the results derived in the Appendix, we write the $A, B$, and $C$ matrices of Eq. (38) to zeroth order as

$$
\begin{aligned}
& \mathrm{A}=\left[\begin{array}{ccc}
A_{00}^{x x} & 0 & 0 \\
0 & A_{00}^{y y} & 0 \\
0 & 0 & 0
\end{array}\right]=\left[\begin{array}{ccc}
c^{2} & 0 & 0 \\
0 & c^{2} & 0 \\
0 & 0 & 0
\end{array}\right], \\
& \mathrm{B}=\left[\begin{array}{ccc}
0 & 0 & 0 \\
0 & 0 & B_{00}^{y z} \\
0 & 0 & 0
\end{array}\right]=\left[\begin{array}{ccc}
0 & 0 & 0 \\
0 & 0 & -i V_{0} 4 \pi n_{0} \Sigma_{\|} \\
0 & 0 & 0
\end{array}\right],
\end{aligned}
$$




$$
\begin{aligned}
\mathrm{C} & =\left[\begin{array}{ccc}
C_{00}^{x x} & C_{00}^{\alpha y} & 0 \\
C_{00}^{y x} & C_{00}^{y y} & C_{00}^{y z} \\
0 & 0 & C_{00}^{z z}
\end{array}\right] \\
& =\left[\begin{array}{ccc}
s^{2}(1+\beta) & s^{2} \beta_{2} & 0 \\
-s^{2} \beta_{2} & s^{2}(1+\beta)+2 \frac{c^{2}}{D^{2}} & -V_{0}\left(\frac{2 z}{d^{2}}\right) 4 \pi n_{0} \Sigma_{\|} \\
0 & 0 & s^{2}\left(1+\beta_{1}\right)+2 \frac{c^{2}}{D^{2}}
\end{array}\right] .
\end{aligned}
$$

Setting the determinant of the single matrix given by the parentheses on the left-hand side of Eq. (38) to zero yields two eigenvalues to zeroth order; namely,

$$
\begin{aligned}
& c^{2}\left(k_{z}^{0}\right)^{2}=-\left(s^{2}(1+\beta)+\frac{c^{2}}{D^{2}}\right)+\sqrt{\frac{c^{4}}{D^{4}}-s^{4} \beta_{2}^{2}}, \\
& c^{2}\left(k_{z}^{1}\right)^{2}=-\left(s^{2}(1+\beta)+\frac{c^{2}}{D^{2}}\right)-\sqrt{\frac{c^{4}}{D^{4}}-s^{4} \beta_{2}^{2}},
\end{aligned}
$$

where $\beta$ and $\beta_{2}$ are functions of $z$ given by

$$
\begin{aligned}
& \beta=\frac{4 \pi \sigma_{\mathrm{P}} e^{-z^{2} / d^{2}}}{s}, \\
& \beta_{2}=\frac{4 \pi \sigma_{\mathrm{H}} e^{-z^{2} / d^{2}}}{s},
\end{aligned}
$$

$s$ is the Laplace transform variable, $D(d)$ is the $1 / e$ halfwidth of the plasma cloud in the $x(z)$ direction, and $\sigma_{\mathrm{P}}$ and $\sigma_{\mathrm{H}}$ are the Pedersen and Hall conductivities, respectively, defined by Eqs. (13) and (14). The corresponding eigenvectors for these two modes are given by

$$
\begin{aligned}
\mathbf{e}^{0} & =\left[\begin{array}{c}
1 \\
\frac{s^{2} \beta_{2}}{c^{2} / D^{2}+\sqrt{\left(c^{4} / D^{4}\right)-s^{4} \beta_{2}^{2}}} \\
0
\end{array}\right], \\
\mathbf{e}^{1} & =\left[\begin{array}{c}
1 \\
s^{2} \beta_{2} \\
c^{2} / D^{2}-\sqrt{\left(c^{4} / D^{4}\right)-s^{4} \beta_{2}^{2}} \\
0
\end{array}\right] .
\end{aligned}
$$

Note that, to zeroth order, the $z$ component of the electric field is zero. This result will be discussed further below.

The eigenvalues obtained above for the zeroth-order system of equations represent dispersion relations for righthand circularly polarized waves [Eq. (57)] and left-hand circularly polarized waves [Eq. (58)]. Plots of the corresponding index of refraction squared $\left(=c^{2} k_{z}^{2} / \omega^{2}\right)$ for each mode as a function of frequency $\omega$, where we have substituted $i \omega$ for the Laplace transform variable $s$, are shown in Figs. 3(a)-3(f) for three positions along the $z$ dimension of the cloud and for two cloud sizes in the $x$ direction. In these figures, the index of refraction squared is normalized to a stated value and the $\log$ of this quantity is plotted along the ordinate. In addition, the sign of this quantity is preserved so that values that fall below zero correspond to evanescent, nonpropagating waves. For these particular plots, we have chosen $\omega_{p e}(z=0)=10 \omega_{c e}, \omega_{c e}=100 \omega_{c i}$, and $D=300$ and $100 \mathrm{~km}$. In the limit of very large plasma clouds $\left(c / D \ll \omega_{c i}\right)$, the dispersion relations take on the usual form expected for right-hand and left-hand circularly polarized waves in a cold plasma imbedded in a background magnetic field (e.g., Krall and Trivelpiece ${ }^{25}$ ). At high frequencies, the left-hand and right-hand modes are both dispersive but become evanescent at frequencies below $\omega_{1}=\left(\omega_{c e} / 2\right)\left(\sqrt{1+4 \omega_{p e}^{2} / \omega_{c e}^{2}}-1\right)$ for the left-hand mode and $\omega_{2}=\left(\omega_{c e} / 2\right)\left(\sqrt{1+4 \omega_{p e}^{2} / \omega_{c e}^{2}}+1\right)$ for the righthand mode, where $\omega_{p e}$ is the electron plasma frequency and $\omega_{c e}$ is the electron cyclotron frequency. The electron and ion cyclotron resonances appear in the dispersion plots as do electron and ion cyclotron waves, whistler waves, and Alfvén waves at low frequencies. As we move to higher $z$ in the cloud, the plasma density decreases and we see $\omega_{1}$ and $\omega_{2}$ shift to lower frequencies. In addition, we find that an evanescent cutoff is introduced at low frequencies in the vicinity of $\omega_{c i}$ as a result of the finite dimension of the radiating plasma antenna. This effect can be understood by first noting that the total wave number $\left(k^{2}=k_{x}^{2}+k_{z}^{2}\right)$ can be found by letting $D \rightarrow \infty$ in Eqs. (57) and (58) with the resuit

$$
\begin{aligned}
& k^{0}=i \frac{s}{c} \sqrt{1+\beta-i \beta_{2}}, \\
& k^{1}=i \frac{s}{c} \sqrt{1+\beta+i \beta_{2}} .
\end{aligned}
$$

The corresponding expressions for the $k_{x}$ components in the zeroth-order limit are then given by

$$
\begin{aligned}
& k_{x}^{0}=\left[\left(\frac{1}{D^{2}}+i \frac{s^{2}}{c^{2}} \beta_{2}\right)-\sqrt{\frac{1}{D^{4}}-\frac{s^{4}}{c^{4}} \beta_{2}^{2}}\right]^{1 / 2}, \\
& k_{x}^{1}=\left[\left(\frac{1}{D^{2}}-i \frac{s^{2}}{c^{2}} \beta_{2}\right)+\sqrt{\frac{1}{D^{4}}-\frac{s^{4}}{c^{4}} \beta_{2}^{2}}\right]^{1 / 2} .
\end{aligned}
$$

At certain frequencies, $k_{x}$ can equal and even exceed $k$ resulting in a negative value for $k_{z}^{2}$ and an evanescent electromagnetic wave. In this regime, the antenna is simply not large enough to radiate at low frequencies. From the plots in Figs. 3(a)-3(f) we see that the cutoff frequency increases with decreasing plasma density and decreasing plasma cloud size. In the limit of large $D, k_{x}$ reduces to $1 / D$ for both modes and the antenna radiates as a smooth aperture at high frequencies with a divergence angle of order $\left(\tan \theta_{D}=k_{x} / k_{z}\right) \theta_{D} \approx \lambda / 2 \pi D$. This result is consistent with the approximation of geometrical optics and is depicted in Fig. 4. Thus, in terms of wave propagation, it is apparent that, for large-enough clouds, the zeroth Hermite polynomial term yields a solution in which the plasma radiates as a smooth aperture at high frequency and cuts off at low frequencies. Higher-order terms should, in general, includc additional radiation effects resulting from any self- 
DISPERSION RELATION $D=300 \mathrm{~km}$
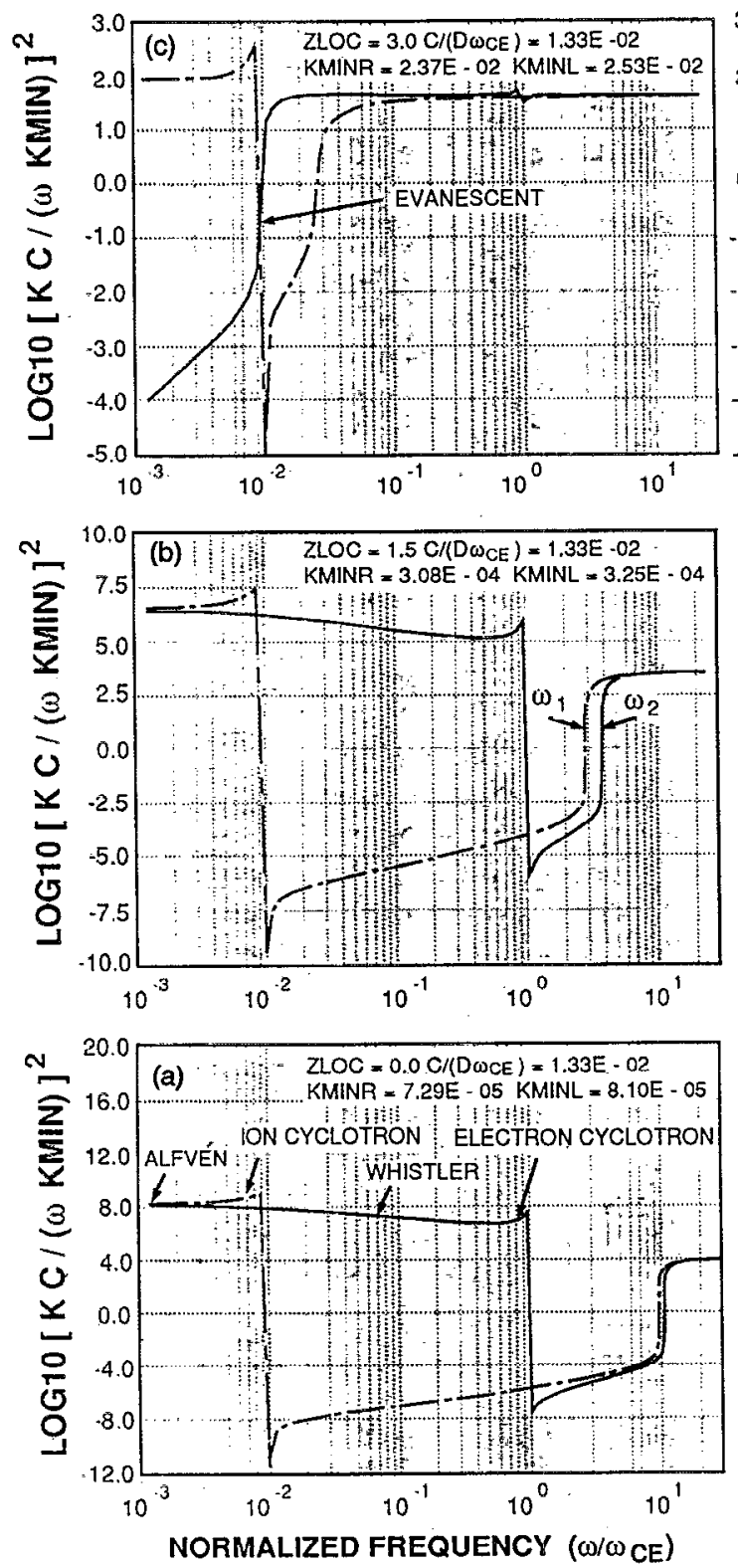

DISPERSION RELATION $D=100 \mathrm{~km}$
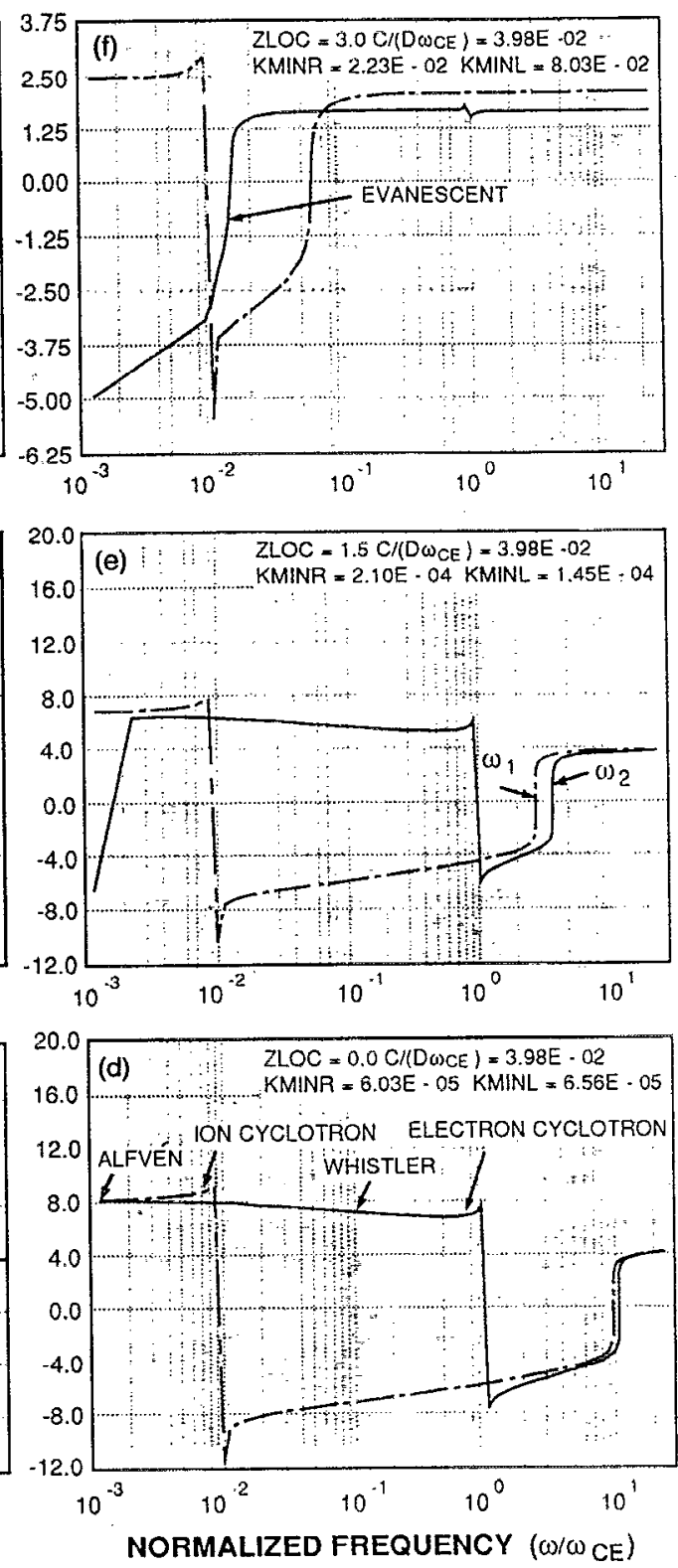

FIG. 3. (a)-(f) The index of refraction squared $\left(=c^{2} k_{z}^{2} / \omega^{2}\right)$ for the two eigenmodes associated with the zeroth-order solution is plotted as a function of frequency $\omega$ at three positions along the $z$ dimension of the cloud and for two cloud dimensions in the $x$ direction. In these figures, the index of refraction squared is normalized to a state value and the log of this quantity is plotted along the ordinate. In addition, the sign of this quantity is preserved so that values that fall below zero correspond to evanescent, nonpropagating waves. For these particular plots we have chosen $\omega_{p e}(z=0)=10 \omega_{c e}$ $\omega_{c e}=100 \omega_{c h}$ and $D=300 \mathrm{~km}[(\mathrm{a})-(\mathrm{c})]$ and $100 \mathrm{~km}[(\mathrm{e})-(\mathrm{f})]$. In the limit of very large plasma clouds $\left(c / D<\omega_{c i}\right)$, the dispersion relations take on the usual form expected for right-hand and left-hand circularly polarized waves in a cold plasma imbedded in a background magnetic field. At high frequencies, the left-hand and right-hand modes are both dispersive but become evanescent at frequencies below $\omega_{1}\left\{=1 / 2 \omega_{c e}\left[\left(1+4 \omega_{p e}^{2} / \omega_{c e}^{2}\right)-1\right]\right\}$ for the left-hand mode and $\omega_{2}\left\{=1 / 2 \omega_{c e}\left[\left(1+4 \omega_{p e}^{2} / \omega_{c e}^{2}\right)+1\right]\right\}$ for the right-hand mode. The electron and ion cyclotron resonances appear in the dispersion plots as do electron and ion cyclotron waves, whistler waves, and Alfvén waves at low frequencies. As we move to higher $z$ in the cloud, the plasma density decreases and we see $\omega_{1}$ and $\omega_{2}$ shift to lower frequencies. In addition, an evanescent cutoff is introduced at low frequencies in the vicinity of $\omega_{c i}$ as a result of the finite dimension of the radiating plasma antenna.

consistent roughness that might develop in the $x$ direction. We note that, at low frequencies $\left(\omega<\omega_{c e}\right)$, the vacuum wavelength of the wave approaches and can even exceed the dimensions of the plasma. However, as seen in Figs. $3(\mathrm{a})-3(\mathrm{f})$, the dielectric constant is large compared to one throughout the low-frequency $\left(\omega<\omega_{c e}\right)$ regime in the central part of the plasma (i.e., at sufficiently high densities). As a result, the dielectric wavelength is much smaller than the plasma dimensions and the assumptions of geometrical optics remain valid. As the plasma density decreases, the 


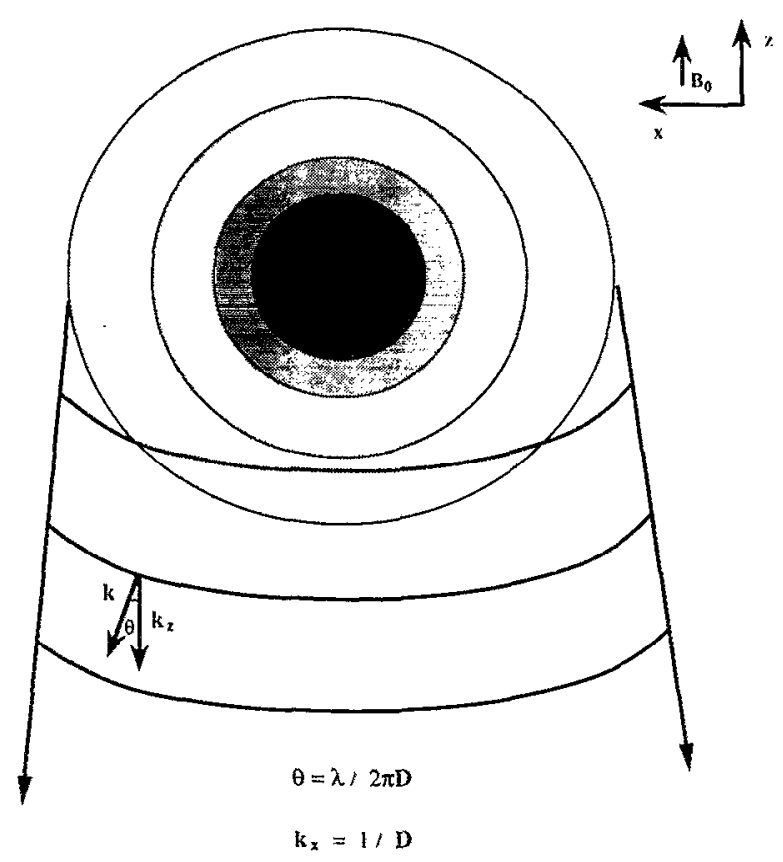

FIG. 4. In the limit of geometrical optics, the plasma radiates as a smooth aperture into a cone with angular divergence of $2 \theta$, where $\theta$ is approximately equal to $\lambda / 2 \pi D$ for large clouds. In this figure, the plasma cloud is depicted as shaded contours that represent the Gaussian plasma density profile and is moving out of the page. The angular spread of the radiation field is shown schematically.

dielectric wavelength approaches the vacuum wavelength and our solution starts to fail at low frequencies. The failure of our solution in this regime is contained in the eigenvectors, which are discussed in detail below.

The eigenvectors also contain interesting information about the nature of the zeroth-order solution. First is the fact that the $z$ component of the electric field is zero. If one considers the picture shown in Fig. 5, it is apparent that

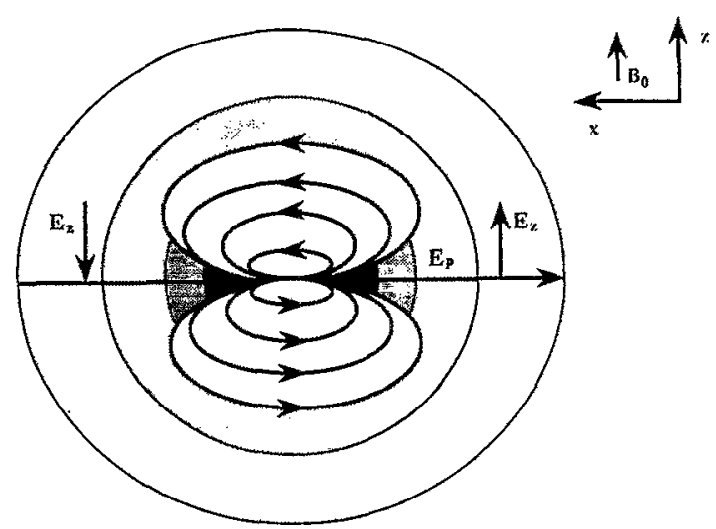

FIG. 5. The charge separation produced in the $x$ direction by the Lorentz force results in a polarization field that closes on itself with a fringing field that has components in the $z$ direction that are asymmetric as a function of $x$. As in Fig. 4, the plasma cloud is depicted as shaded contours that represent the Gaussian plasma density profile and is moving out of the page. The fringing field associated with polarization of the plasma is shown schematically. the charge separation produced in the $x$ direction by the Lorenz force results in a polarization field that closes on itself with a fringing field that has components in the $z$ direction that are asymmetric as a function of $x$. Because the zeroth-order solution is defined in terms of even functions of $k$, the inverse Fourier transform is even in $x$, and therefore to this order the $z$ electric field is zero. Second, it can be shown (numerically) that $k_{z}^{0}\left(s^{*}\right)=-\left[k_{z}^{1}(s)\right]^{*}$ and $\mathbf{e}_{0}\left(s^{*}\right)=-\left[\mathbf{e}_{1}(s)\right]^{*}$. These two conditions together guarantee that our final solution will satisfy causality [written as $\left.\mathbf{E}^{*}\left(s^{*}\right)=\mathbf{E}(s)\right]$ but are also consistent with the fact that a negatively propagating right-hand polarization wave looks like a positively propagating left-hand polarization wave. Third, we find that the eigenvectors are functions of $z$. In order to be consistent with the approximation of geometrical optics, it is necessary that these coefficients vary slowly compared to the vacuum wavelength of waves generated by the plasma. To examine the consistency of this approximation, we have plotted in Figs. 6(a)-6(f) the $y$ component of the eigenvectors as a function of $z$ for various frequencies. In all cases, we find that these coefficients are constant over a large part of the cloud but that the approximation begins to break down at the edge of the plasma, as noted previously.

The zeroth-order Green's function is derived based on the procedure outlined in Sec. III B with the result

$$
\begin{aligned}
& g_{0 n}^{x>}=-\frac{H_{0}\left(k^{\prime} D / 2\right)}{(2 \sqrt{\pi} / D) c^{2}} \\
& \cdot\left(\frac{\left(c^{2} / D^{2}+\sqrt{c^{4} / D^{4}-s^{4} \beta_{2}^{2}}\right)}{4 k_{z}^{n} \sqrt{c^{4} / D^{4}-s^{4} \beta_{2}^{2}}} e^{i \int_{z^{\prime}}^{0} k_{z^{\prime \prime}}^{n} d z^{\prime \prime}}\right), \\
& g_{0 n}^{y>}=-\frac{H_{0}\left(k^{\prime} D / 2\right)}{(2 \sqrt{\pi} / D) c^{2}} \cdot\left(\frac{s^{2} \beta_{2}}{4 k_{z}^{n} \sqrt{c^{4} / D^{4}-s^{4} \beta_{2}^{2}}} e^{i \int_{z^{\prime}}^{0} k_{z^{\prime \prime}}^{n} d z^{\prime \prime}}\right),
\end{aligned}
$$

where $n$ takes on the values 0 and 1 and where the $h_{0 n}^{x<}$ and $h_{0 n}^{y<}$ are identical to their corresponding $g$ counterparts (with $>$ replaced by $<$ ) except for a minus sign in the exponential. The final solution for the normalized (to $\left.V_{0} B_{0} / c\right)$ electric field is then obtained from Eqs. (35) and (53) and is given by

$$
\begin{aligned}
\widetilde{\mathbf{E}}(s, x, z)= & e^{-x^{2} / D^{2}}\left(\int _ { - \infty } ^ { z } d z ^ { \prime } \frac { s } { c ^ { 2 } } \left[\mathbf{e}_{0} \alpha_{0}\left(s, z^{\prime}\right) e^{i \int_{z^{\prime}}^{z} k_{z^{\prime \prime}}^{0} d z^{\prime \prime}}\right.\right. \\
& \left.\left.+\mathbf{e}_{1} \alpha_{1}\left(s, z^{\prime}\right) e^{i \int_{z^{\prime}}^{z}, k_{z^{\prime \prime}}^{1} d z^{\prime \prime}}\right]\right) \\
& +e^{-x^{2} / D^{2}}\left(\int _ { z } ^ { \infty } d z ^ { \prime } \frac { s } { c ^ { 2 } } \left[\mathbf{e}_{0} \alpha_{0}\left(s, z^{\prime}\right) e^{-i \int_{z^{\prime}}^{z} k_{z^{\prime \prime}}^{0} d z^{\prime \prime}}\right.\right. \\
& \left.\left.+\mathbf{e}_{1} \alpha_{1}\left(s, z^{\prime}\right) e^{-i \int_{z^{\prime}}^{z}, k_{z^{\prime \prime}}^{1} d z^{\prime \prime}}\right]\right)
\end{aligned}
$$

where the coefficients $\alpha_{0}$ and $\alpha_{1}$ are defined as

$$
\alpha_{0}=\frac{s^{2} \beta_{2}^{2}+\beta\left(c^{2} / D^{2}+\sqrt{c^{4} / D^{4}-s^{4} \beta_{2}^{2}}\right)}{4 i k_{z^{\prime}}^{0} \sqrt{c^{4} / D^{4}-s^{4} \beta_{2}^{2}}}
$$



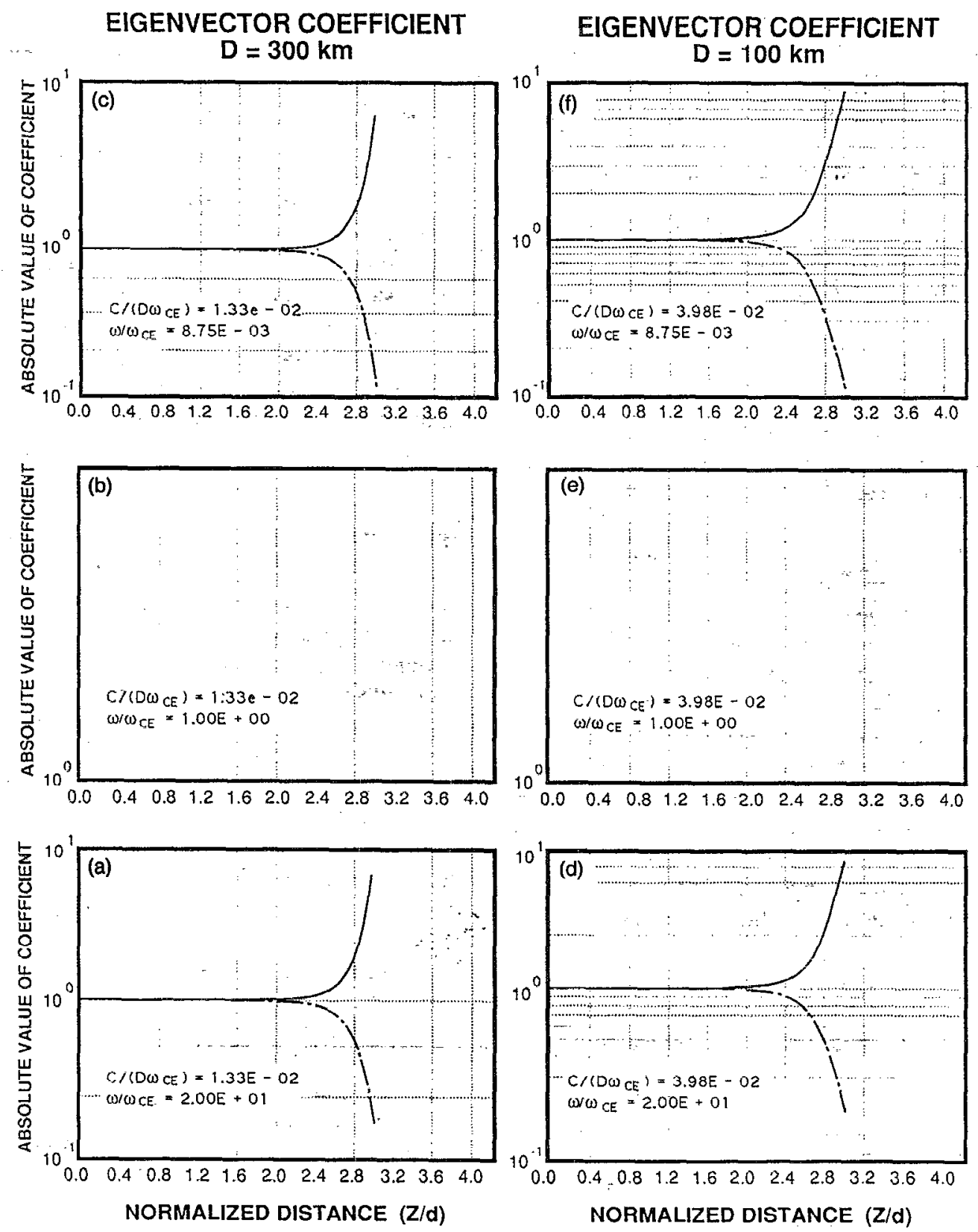

FIG. 6. The $y$ component of the two eigenvectors is plotted as a function of normalized distance $z / d$ for three frequencies $\left[\omega=20 \omega_{c e}(a) ; \omega=\omega_{c e}(b)\right.$; and $\omega=0.00875 \omega_{c e}$ (c)] and for the two cloud dimensions in the $x$ direction $[D=300 \mathrm{~km}$ (a) $-(\mathrm{c})$ and $D=100 \mathrm{~km}$ (d) $-(\mathrm{f})]$.

$$
\alpha_{1}=\frac{s^{2} \beta_{2}^{2}+\beta\left(c^{2} / D^{2}+\sqrt{c^{4} / D^{4}-s^{4} \beta_{2}^{2}}\right)}{4 i k_{z^{\prime}}^{1} \sqrt{c^{4} / D^{4}-s^{4} \beta_{2}^{2}}}
$$

and where we have performed the inverse Fourier transform in $x$.

From Eq. (69), it is apparent that the solution for the fields at position $z$ and for a given frequency $s$ is determined by computing the contribution that left-hand and righthand polarization waves launched at each position $z^{\prime}$ make to the amplitude at $z$ and summing over all $z^{\prime}$. The contribution of $z$ of the waves generated at $z^{\prime}$ is given by a local frequency- and position-dependent amplitude times the exponential of the accumulated phase change of the wave between $z$ and $z^{\prime}$, in a way that is consistent with geometrical optics. The denominator inside the $z^{\prime}$ integral has zeros at the electron and ion cyclotron frequencies and at frequencies where $k_{z}$ goes to zero. These frequencies correspond to the natural modes of oscillation of the plasma. When $k_{z}$ is set equal to zero, the wave equations (17)-(19) collapse to a set of equations that describe the local formation of a polarization field (driven by the Lorentz force) in the $x$ direction and that include a coupling to an induction field in the $y$ direction, permitted by the spatial variations in $x$ leading to a $d B_{z} / d x$ and by the presence of currents in the $y$ direction. Thus, by taking $k_{z}$ equal to zero, we define the local amplitude of the currents that are driven by the 
Lorentz force and their natural modes of oscillation. Clearly, the dominant contribution to the integral comes from these modes and the solution is therefore characterized by waves that are launched at the corresponding frequencies and that propagate through the plasma in a way consistent with geometrical optics. To obtain the timedependent evolution of the plasma cloud it is necessary to perform the inverse Laplace transform of Eq. (69). The inverse transform has been calculated numerically for various plasma parameters and the results are described in a companion publication.

\section{SUMMARY}

The early-time ( $<100$ ion gyroperiods) evolution of plasmas moving across a background magnetic field has been addressed with a two-dimensional model in which a plasma cloud is assumed to have formed instantaneously with a velocity $V_{0}$ across a uniform background magnetic field and with a Gaussian density profile in the two dimensions perpendicular to the direction of motion. This model treats both the early-time dynamics associated with the formation of the polarization field and the generation and propagation of electromagnetic waves. Several approximations are made in our description of the plasma dynamics and our treatment of the propagation of electromagnetic waves. First, the plasma is assumed to have a Maxwellian velocity distribution function and only the moments of the distribution function (density, mean velocity, and temperature) are evolved in time (by means of the fluid equations). As a result, we do not include the effects of kinetic instabilities or resonant wave-particle interactions. Second, the plasma dimensions are assumed to be much larger than an ion gyroradius and we therefore ignore the additional current systems and corresponding electromagnetic radiation that arise in connection with momentum and energy transport processes driven by density, velocity, and temperature gradients. Third, the production rate of additional plasma and plasma momentum resulting, for example, from photoionization of neutrals that are moving across the magnetic field is assumed to be small compared to an ion gyrofrequency. This effect can therefore be neglected over time scales small compared to an ion gyroperiod, however, in treating the self-consistent evolution of the plasma and the radiation field over longer time scales it is important to include it. Fourth, the initial dimensions of the plasma are assumed to evolve in time in a way that is consistent with the neglect of pressure and velocity gradients. In general, this effect can be omitted over short time scales, however, plasma dimensions are observed to change over longer time scales (c.g., in active experiments) and, as in the case of plasma production, this effect must be included in order to treat the self-consistent evolution of the plasma and the radiation field over the longer times of interest. Fifth, the plasma is assumed to be collisionless. Sixth, the effects of a background plasma are omitted. Depending on its density relative to that of the plasma cloud, the background plasma can both neutralize the polarization field and affect the propagation of electromagnetic radiation launched by the cloud. It will be important to in- clude the background in future studies. Seventh, the dimensions of the plasma cloud are assumed to be large compared to the vacuum wavelength or dielectric wavelength of radiation launched during the early phases of evolution of the plasma. This approximation allows us to treat the propagation of electromagnetic radiation in the limit of geometrical optics. In addition, because the primary source of radiation is the oscillating polarization field perpendicular to the magnetic field and because the plasma dimensions are large, the electromagnetic waves propagate primarily along the magnetic field. This fact allows for an expansion of the fields in terms of Hermite polynomials, which as the order of the expansion increases, incorporates additional effects associated with small and smaller-scale structures in the direction of the polarization field.

With these approximations we were able to obtain an analytic solution to the two-dimensional, coupled fluid [Eqs. (7)-(9)] and Maxwell's equations [Eq. (20)]. A Fourier transform taken in one spatial dimension and a Laplace transform in time yields a set of integrodifferential equations [Eqs. (17)-(19) and Eq. (23)] for the electromagnetic fields. These equations are then converted to a coupled set of second-order, ordinary differential equations by expanding the fields in terms of Hermite polynomials [see the ansatz Eqs. (35) and (46)] and making use of the orthogonality condition. The Green's function technique is then used together with a specification of the initial conditions for the plasma and field quantities and their temporal derivatives over all space to find the final solution. While the procedure for obtaining the solution to any order in the Hermite polynomial expansion is provided, a general solution cannot be written explicilly. Instead, the specific solution for the zeroth-order term in the Hermite polynomial expansion is derived. In general, the results indicate that, to zeroth order, the plasma cloud behaves like a large dipole antenna oriented in the direction of the polarization field which is set up by the initial cross-field motion of the electrons and ions and which oscillates at frequencies defined by the normal modes of the system; namely, where the $z$ wave number (eigenvalue) vanishes. These frequencies are density dependent and are highest in the center of the plasma cloud. The high-frequency electromagnetic radiation emitted by the corresponding current systems propagate through the cloud, interacting with the intervening plasma, and eventually into vacuum. At low frequencies, the dipole antenna is too small to radiate, and this radiation becomes trapped inside the plasma. The magnitude of the radiation field and the amount of plasma momentum and energy carried away by and stored instantaneously in the fields depends on the relative magnitude of certain plasma parameters. These issues will be addressed in a companion paper where results for specific cloud parameters are presented and where scaling laws for the magnitude of the fields and the slowing down of the plasma cloud are derived.

The present analysis has direct application to the crossfield motion of plasmas in experiments where the background plasma density is small compared to the cloud density and where the cloud dynamic $\beta$ is small (e.g., 
magnetospheric chemical releases). The general application to active experiments and to space and astrophysical plasmas, however, will necessitate the inclusion of additional effects. First, the Hermite polynomial expansion will have to be taken to first order so that the $z$ electric field can be determined. Second, the effects of a background plasma will have to be included. Third, plasma density and momentum production (as obtained, for example, in active experiments) will have to be modeled in order to treat the late time evolution of the plasma and electromagnetic radiation self-consistency. Finally, expansion of the plasma dimensions with time (also obtained in active experiments) can also affect the self-consistent evolution of the plasma and radiation fields over long time scales. These issues will be addressed in future publications.

\section{ACKNOWLEDGMENTS}

The authors would like to thank Dr. David J. Simons for many helpful discussions and for his support and encouragement throughout this effort.

Research at Los Alamos was performed under the auspices of the U. S. Department of Energy and was supported in part by the Office of Space Science and Applications (OSSA) of the National Aeronautics and Space Administration (NASA). This work was supported at the University of Michigan by NASA Grants No. NAGW-2162 and No. NAGW-1619 and by the National Science Foundation (NSF) under Contract No. ATM-9114409.

\section{APPENDIX: DERIVATION OF MATRIX ELEMENTS}

Equation (38) was obtained by substituting the ansatz Eq. (35) into the electromagnetic wave equations, Eqs. (17)-(19), and dotting the resulting equations with a Hermite polynomial of order $l$. The dot product referred to here and in the text between the Hermite polynomial and a given function $\Psi(k)$ is defined as

$$
H_{l} \cdot \Psi(k)=\int_{-\infty}^{\infty} d k H_{l}(k) \Psi(k) e^{-k^{2}} .
$$

In deriving the matrix elements for the matrices $A, B$, and $C$ with the procedure outlined above, it is necessary to perform certain integrals over $k$; namely,

$$
\begin{aligned}
& \int_{-\infty}^{\infty} d k k H_{n}\left(\frac{k D}{2}\right) H_{l}\left(\frac{k D}{2}\right) e^{-k^{2} D^{2} / 4} \\
& =\frac{2 \pi^{1 / 2}}{D^{2}} 2^{l}\left[2(l+1) ! \delta_{n, l+1}+l(l-1) ! \delta_{n, l-1}\right], \\
& \int_{-\infty}^{\infty} d k k^{2} H_{n}\left(\frac{k D}{2}\right) H_{l}\left(\frac{k D}{2}\right) e^{-k^{2} D^{2} / 4} \\
& =\frac{4 \pi^{1 / 2}}{D^{3}}\left[2^{l+1}(l+2) ! \delta_{n, l+2}+(2 l+1) 2^{l} l ! \delta_{n, l}\right. \\
& \left.+2 l(l-1) 2^{l-2}(l-2) ! \delta_{n, l-2}\right], \\
& \int_{-\infty}^{\infty} d k \int_{-\infty}^{\infty} \frac{d k^{\prime}}{2 \pi} e^{-\left(k-k^{\prime}\right)^{2} D^{2} / 4} H_{n} \\
& \times\left(\frac{k^{\prime} D}{2}\right) H_{l}\left(\frac{k D}{2}\right) e^{-k^{2} D^{2} / 4} \\
& =\left\{\begin{array}{l}
\frac{2}{D^{2}}\left(\frac{1}{2}\right)^{n / 2} 2^{3 n / 2} \frac{n}{[(l-n) / 2] !} \quad 1 \geqslant n \\
0 \quad 1<n
\end{array}\right\}, \\
& \int_{-\infty}^{\infty} d k k \int_{-\infty}^{\infty} \frac{d k^{\prime}}{2 \pi} e^{-\left(k-k^{\prime}\right)^{2} D^{2} / 4} H_{n} \\
& \times\left(\frac{k^{\prime} D}{2}\right) H_{l}\left(\frac{k D}{2}\right) e^{-k^{\prime 2} D^{2} / 4} \\
& =\left\{\begin{array}{ll}
\frac{2}{D^{3}} 2^{n} \frac{(l+1) !}{[(l-n+l) / 2] !} \quad l \geqslant n-1 \\
0 \quad l<n-1
\end{array}\right\} \\
& +\left\{\begin{array}{lll}
\frac{2}{D^{3}} & 2^{n+1} \frac{(l-1) !}{[(l-n-1) / 2] !} l \\
0 & l<n+1
\end{array}\right\},
\end{aligned}
$$

where the results for these integrals were taken from Gradshteyn and Ryzhik ${ }^{26}$ (pp. 837-839). With these results, the matrix elements are readily found to be

$$
\begin{aligned}
& A_{l n}^{x x}=A_{l n}^{y \bar{y}}=\frac{2 \pi^{1 / 2}}{D^{2}} 2^{I l l} \delta_{l, n}, \\
& A_{l n}^{x y}=A_{l n}^{x z}=A_{l n}^{y x}=A_{l n}^{y z}=A_{l n}^{z x}=A_{l n}^{z y}=A_{l n}^{z z}=0,
\end{aligned}
$$

$$
\begin{aligned}
& B_{l n}^{x z}=-B_{l n}^{z x}=\frac{2 \pi^{1 / 2} c^{2}}{D^{2}} 2^{l}\left[2(l+1) ! \delta_{n, l+1}+l(l-1) ! \delta_{n, l-1}\right] \\
& B_{l n}^{y z}=\left\{\begin{array}{cc}
-i V_{0} \frac{2 \pi^{1 / 2}}{D} 4 \pi n_{0} e^{-z^{2} / d^{2}} \Sigma_{\|} \frac{2^{n} l !}{[(l-n) / 2] !} & 1>n, l+n=\text { even } \\
0 & 1<n, l+n=\text { odd }
\end{array}\right\}
\end{aligned}
$$




$$
\begin{aligned}
& C_{l n}^{x x}=\left\{\begin{array}{cc}
\frac{2 \pi^{1 / 2}}{D} s^{2} 2^{l} l \| \delta_{n, l}+\frac{2 \pi^{1 / 2}}{D} s 4 \pi n_{0} e^{-z^{2} / d^{2}} \Sigma_{\mathrm{P}} \frac{2^{n} l !}{[(l-n) / 2] !} \quad l \geqslant n, l+n=\text { even } \\
0 \quad l<n, l+n=\text { odd }
\end{array}\right\} \\
& C_{l n}^{x y}=\left\{\begin{array}{cc}
\frac{2 \pi^{1 / 2}}{D} s 4 \pi n_{0} e^{-z^{2} / d^{2}} \Sigma_{\mathrm{H}} \frac{2^{n} l !}{[(l-n) / 2] !} l \geqslant n, l+n=\mathrm{even} \\
0 \quad l<n, l+n=\mathrm{odd}
\end{array}\right\}, \\
& C_{l n}^{y x}=\left\{\begin{array}{ccc}
-\frac{2 \pi^{1 / 2}}{D} s 4 \pi n_{0} e^{-z^{2} / d^{2}} \Sigma_{\mathrm{H}} \frac{2^{n} 1 !}{[(1-n) / 2] !} & 1 \geqslant n, 1+n=\text { even } \\
0 & 1<n, 1+n=\text { odd }
\end{array}\right\} \\
& +\left\{\begin{array}{cc}
i V_{0} \frac{2 \pi^{1 / 2}}{D^{2}} 4 \pi n_{0} e^{-z^{2} / d^{2}} \Sigma_{\mathrm{P}} \frac{2^{n}(l+1) !}{[(l-n+1) / 2] !} l \geqslant n-1 \\
0 & 0
\end{array}\right\}
\end{aligned}
$$

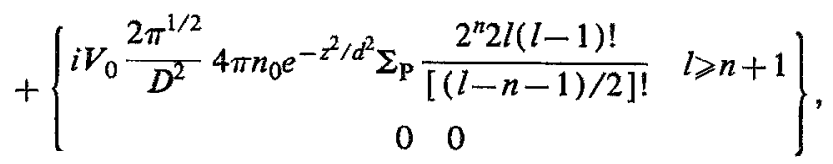

$$
\begin{aligned}
& C_{l n}^{y y}=\frac{2 \pi^{1 / 2}}{D} s^{2} 2^{l} l ! \delta_{n, l}+\frac{2 \pi^{1 / 2}}{D} \frac{2 c^{2}}{D^{2}}\left[2^{l+1}(l+2) ! \delta_{n, l+2}+(2 l+1) 2 l n \delta_{n, l}+2 l(l-1) 2^{l-2}(l-2) ! \delta_{n, l-2}\right] \\
& +\left\{\begin{array}{cc}
\frac{2 \pi^{1 / 2}}{D} s 4 \pi n_{0} e^{-z^{2} / d^{2}} \Sigma_{\mathrm{P}} \frac{2^{n} l !}{[(l-n) / 2] !} l \geqslant n, l+n=\text { even } \\
0 \quad l<n, l+n=\text { odd }
\end{array}\right\}
\end{aligned}
$$

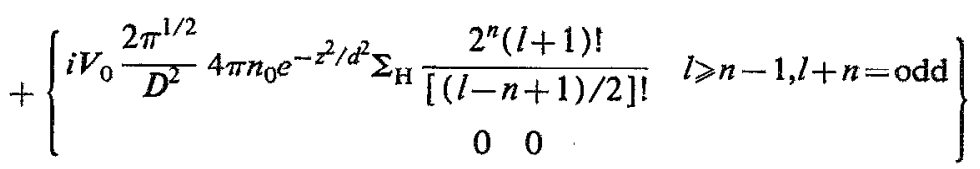

$$
\begin{aligned}
& +\left\{\begin{array}{cc}
i V_{0} \frac{2 \pi^{1 / 2}}{D^{2}} 4 \pi n_{0} e^{-z^{2} / d^{2}} \Sigma_{\mathrm{H}} \frac{2^{n} 2 l(l-1) !}{[(l-n-1) / 2] !} & l \geqslant n+1, l+n=\text { odd } \\
0 & 0
\end{array}\right\} \text {, } \\
& C_{l n}^{\mathrm{gz}}=\left\{\begin{array}{c}
-V_{0}\left(\frac{2 z}{d^{2}}\right) \frac{2 \pi^{1 / 2}}{D} 4 \pi n_{0} e^{-z^{2} / d^{2}} \Sigma_{\|} \frac{2^{n} \eta}{(l-n) / 2 !} \\
0 \quad l<n, l+n=\text { odd }
\end{array}\right\} \\
& C_{l n}^{c z}=\frac{2 \pi^{1 / 2}}{D} s^{2} 2^{l} l ! \delta_{n, l}+\frac{2 \pi^{1 / 2}}{D} \frac{2 c^{2}}{D^{2}}\left[2^{l+1}(l+2) ! \delta_{n, l+2}+(2 l+1) 2^{l} l ! \delta_{n, l}+2 l(l-1) 2^{l+2}(l-2) ! \delta_{n, l+2}\right] \\
& +\left\{\begin{array}{cc}
\frac{2 \pi^{1 / 2}}{D} s 4 \pi n_{0} e^{-z^{2} / d^{2}} \Sigma_{\|} & \frac{2^{n} l !}{[(l-n) / 2] !} l \geqslant n, l+n=\text { even } \\
0 & l<n, l+n=\text { odd }
\end{array}\right\},
\end{aligned}
$$

$$
C_{l n}^{x z}=C_{l n}^{\alpha x}=C_{l n}^{a y}=0
$$

$$
S_{l}^{z}=0
$$

Similarly, the vector elements of the source term $\mathbf{S}$ dotted with $H_{I}$ become,

where we have obtained these results after division of our equations by $V_{0} B_{0} / c$ so that the source terms presented here are normalized to this factor.

$S_{l}^{y}=8 \pi^{2} n_{0} e^{-z^{2} / d^{2}} \Sigma_{\mathrm{H}} \delta_{l 0}-i \frac{16 V_{0}}{D s} \pi^{2} n_{0} e^{-z^{2} / d^{2}} \Sigma_{\mathrm{P}} \delta_{l 1}$,

'J. Zinn, H. Hoerlin, and A. G. Petschek, Radiation Trapped in the Earth's Magnetospheric Field, edited by B. M. McCormac (Reidel, Hingham, MA, 1966), p. 671 .

${ }^{2} J$. E. Borovsky, Phys. Fluids 30, 2518 (1987). 
${ }^{3}$ M. Scholer, Planet. Space Sci. 18, 977 (1970).

${ }^{4}$ C. K. Goertz and R. W. Boswell, J. Geophys. Res. 84, 7239 (1979):

${ }^{5}$ G. F. Nalesso and A. R. Jacobson, J. Geophys. Res. 93, 5794 (1988).

${ }^{6}$ G. F. Nalesso and A. R. Jacobson, J. Geophys. Res. 93, 5803 (1988).

${ }^{7}$ C. K. Goertz and P. A. Deift, Planet. Space Sci. 21, 1399 (1973).

${ }^{8} \mathrm{~J}$. W. Belcher, C. K. Goertz, J. D. Sullivan, and M. H. Acuna, J. Geophys. Res, 86, 8505 (1981).

${ }^{9}$ Y. P. Maltsev, W. B. Lyatsky, and A. M. Lyatskaya, Planet Space Sci. 25, 53 (1977).

${ }^{10} \mathrm{G}$. Haerendel, High Latitude Space Plasma Physics- (Plenum, New York, 1982).

${ }^{11}$ R. L. Lysak, J. Geophys. Res. 91, 7047 (1986).

${ }^{12}$ M. Galvez, Phys. Fluids 30, 2729 (1987).

${ }^{13} \mathrm{M}$. Galvez and C. Barnes, Phys. Fluids 31, 863 (1988).

${ }^{14}$ M. Galvez, S. P. Gary, C. Barnes, and D. Winske, Phys. Fluids 31, 1554 (1988).

${ }^{15}$ M. Galvez, G. Gisler, and C. Barnes, Phys. Fluids B 1, 2516 (1989).

${ }^{16}$ R. W. Schunk and E. P. Szuszczewics, J. Geophys. Res. 93, 12901 (1988).
${ }^{17}$ D. Winske, J. Geophys. Res. 93, 2539 (1988).

${ }^{18}$ W. A. Livesey and P. L. Pritchett, Phys. Fluids B 1, 914 (1989).

${ }^{19}$ A. G. Sgro, S. P. Gary, and D. S. Lemons, Phys. Fluids B 1, 1890 (1989).

${ }^{20}$ K. Papadopoulos, A. Mankofsky, R. C.: Davidson, and A. T. Drobot, , Phys. Fluids B 3, 1075 (1991).

${ }^{21}$ A. B. Hassam and J. D. Huba, Phys. Fluids 31, 318 (1988).

${ }^{22}$ J. D. Huba, J. G. Lyon, and A. B. Hassam, Phys. Rev. Lett. 59, 2971 (1987).

${ }^{23}$ M. Abramowitz and I. A. Stegun, Handbook of Mathematical Functions (U. S. Department of Commerce, National Bureau of Standards, Washington, DC, 1964), Applied Mathematical Series 55, Library of Congress Catalog Card No. 64-60036.

${ }^{24} \mathrm{M}$. Born and E. Wolf, Principles of Optics (Pergamon, Elmsford, New York; 1959)

${ }^{25}$ N. - A. Krall and A. W. Trivelpiece, Principles of Plasma Physics (McGraw-Hill, New York, 1973).

${ }^{26}$ I. S. Gradshteyn and I. M. Ryzhik, Table of Integrals Series and Products (Academic, New York, 1965). 